\title{
Dynamical backreaction of relic gravitons
}

\author{
Massimo Giovannini* \\ Centro “Enrico Fermi", Compendio del Viminale, Via Panisperna 89/A, 00184 Rome, Italy \\ and Department of Physics, Theory Division, CERN, 1211 Geneva 23, Switzerland
}

(Received 15 January 2006; published 5 April 2006)

\begin{abstract}
The dynamical effects of the tensor modes of the geometry are investigated in the context of curvature bounces. Since the bouncing behavior implies sharp deviations from a radiation-dominated evolution, significant backreaction effects of relic gravitons may be expected at short wavelengths. After developing a general iterative framework for the calculation of dynamical backreaction effects, explicit analytical and numerical examples are investigated for different parametrizations of the energy-momentum pseudotensor(s) of the produced gravitons. The reported results suggest that dynamical backreaction effects are a necessary ingredient for a consistent description of bouncing models at late times.
\end{abstract}

DOI: 10.1103/PhysRevD.73.083505

\section{INTRODUCTION}

Relic gravitons are copiously produced in the early Universe due to the pumping action of the background geometry [1,2]. If a quasi-de Sitter phase of expansion is followed by a radiation-dominated phase, the logarithmic energy spectrum (in units of the critical energy density), customarily denoted by $\Omega_{\mathrm{gw}}(\nu)$, is quasiflat [3] (see also [4-6]) for present (physical) frequencies $\nu$ ranging between $10^{-16} \mathrm{~Hz}$ and, approximately, $100 \mathrm{MHz}$. The transition from the radiation-dominated to the matter epoch produces an infrared branch where $\Omega_{\mathrm{gw}} \sim \nu^{-2}$ between $10^{-18} \mathrm{~Hz}$ and $10^{-16} \mathrm{~Hz}$ [7-9].

In pre-big bang models [10,11], the spectrum of relic gravitons is far from scale-invariant. The spectral slope of $\Omega_{\mathrm{gw}}(\nu)$ (for frequencies larger than $10^{-16} \mathrm{~Hz}$ ) is violet, i.e. $\Omega_{\mathrm{gw}} \sim \nu^{\gamma}$ with $\gamma>1[5,12]$ (see also [13] and, in a complementary perspective, [14]). Minimal pre-big bang models are characterized by a slope $\gamma=3$ that become progressively less steep as the frequency increases in the $\mathrm{kHz}$ and $\mathrm{MHz}$ region.

Depending on the features of the cosmological model, the energy density of relic gravitons may affect the dynamics and change the time evolution of the scale factor as well as of the other homogeneous quantities. If the equation of state of the sources of the background geometry is

\footnotetext{
*Electronic address: massimo.giovannini@cern.ch

${ }^{1}$ It is relevant to remark that the authors of Refs. [16,17] choose to compute the energy and pressure density of the produced particles by means of a perturbative expansion whose small parameter is the deviation from conformal coupling $(\mid \xi-$ $1 / 6$ in the notations of [17]). This approach (also discussed in $[18,19])$ may be applied, with some caveats, in the minimally coupled case (corresponding to $\xi \rightarrow 0$ ) where, it could be argued that the expansion parameter does not exceed 1 . In the present investigation this expansion will not be used since the coupling of relic gravitons to the background geometry is not close to conformal. Furthermore, the expansion in $|\xi-1 / 6|$ may partially break down during the bouncing regime. When needed, numerical techniques will be employed.
}

PACS numbers: 98.80.Cq, 04.62.+v, 11.25.-w, 98.80.Bp

stiffer than radiation (i.e. $p=w \rho$ with $w>1 / 3$ ) the relativistic gravitons of short wavelengths can change the dynamical evolution, as it was noticed in the context of the stiff model of Zeldovich [2].

When a stiff phase follows an inflationary stage of expansion [15] (see also $[16,17]^{1}$ ) the backreaction effects of the produced gravitons set a limit on the possible duration of the stiff (post-inflationary) phase. This aspect is relevant in different situations and, for instance, in the context of quintessential inflationary models [20] where $\Omega_{\mathrm{gw}} \propto \nu$ for frequencies larger than the $\mathrm{mHz}$ [21-23] (see also [24-27] for possible detection strategies). Blue spectral slopes have been also derived in the context of quintessential models on the brane [28,29] (see also [30]). Constraints arising from the production of relic gravitons in quintessential models have been discussed in [31].

Defining as $\tau$ the conformal time coordinate and $k$ as the comoving wave number, two physically different regimes appear naturally in the problem. If $k \tau \gg 1$ (short wavelength limit) the Fourier modes of the tensor fluctuations of the geometry are said to be superadiabatically amplified. In the opposite regime, i.e. $k \tau \ll 1$ (long wavelength limit) the tensor modes are oscillating. In the short wavelength limit the effective equation of state obeyed by the relic gravitons is radiative, i.e. $\left\langle p_{\mathrm{gw}}\right\rangle=\left\langle\rho_{\mathrm{gw}}\right\rangle / 3$ where $\left\langle p_{\mathrm{gw}}\right\rangle$ and $\left\langle\rho_{\mathrm{gw}}\right\rangle$ correspond to the averaged pressure and energy densities. In this case, $\left\langle\rho_{\mathrm{gw}}\right\rangle \sim a^{-4}$, where $a(\tau)$ is the scale factor. In the long wavelength limit the effective equation of state may depend on the salient features of the background evolution. For instance, during the transition from a quasi-de Sitter stage of expansion to a radiation-dominated stage, the modes of long wavelengths are compatible with an effective equation of state $\left\langle p_{\mathrm{gw}}\right\rangle=-\left\langle\rho_{\mathrm{gw}}\right\rangle / 3$ implying that $\left\langle\rho_{\mathrm{gw}}\right\rangle \sim a^{-2}$. In this limit the system behaves, effectively, as a generalized fluid dominated by the spatial gradients of the tensor modes of the geometry.

While the conclusions mentioned in the previous paragraph must rely on specific definitions of the energy and pressure densities of the relic gravitons, it is well known 
that, in general, it is impossible to assign a localized energy density to the gravitational field [32]. This caveat does not exclude the possibility of adopting consistent frameworks for the analysis of a gravitational energy-momentum pseudotensor.

To be more specific, the tensor modes of the geometry can be characterized by a rank-two tensor $h_{i j}$ defined in the three spatial dimensions (that will taken to be flat), i.e.

$$
\begin{aligned}
d s^{2} & =a^{2}(\tau)\left[d \tau^{2}-\left(\delta_{i j}+h_{i j}\right) d x^{i} d x^{j}\right], \\
h_{i}^{i} & =\partial_{i} h_{j}^{i}=0,
\end{aligned}
$$

where $\delta_{i j}$ is Kroeneker symbol and $h_{i j}$, being traceless and divergenceless, carries two independent degrees of freedom that correspond to the two polarizations of a gravitational wave in a conformally flat Friedmann-RobertsonWalker (FRW) background. ${ }^{2}$ The simplest way of estimating the impact of the created gravitons on the background dynamics is by computing the lowest-order nonlinear corrections to the Einstein tensor

$$
G_{\mu}^{\nu}=R_{\mu}^{\nu}-\frac{1}{2} \delta_{\mu}^{\nu} R
$$

where $R_{\mu}^{\nu}$ and $R$ are, respectively, the Ricci tensor and the Ricci scalar. The nonlinear corrections to the Einstein tensor, will consist, to lowest order, of quadratic combinations of $h_{i j}$ that can be formally expressed as ${ }^{3}$

$$
\ell_{\mathrm{P}}^{2} \mathcal{T}_{\mu}^{\nu}=-\delta_{\mathrm{t}}^{(2)} G_{\mu}^{\nu},
$$

where the superscript at the right-hand side denotes the second-order fluctuation of the corresponding quantity while the subscript refers to the tensor nature of the fluctuations. This procedure is essentially the one described in $[33,34]$ and has been reexplored, in a related context, by the authors of Refs. [35,36] mainly in connection with conventional inflationary models where the Universe is always expanding ${ }^{4}$

In [38] (see also [39]) a complementary perspective was invoked and it was observed that, by perturbing the Einstein-Hilbert action to second order in the amplitude of the tensor modes of the geometry in a FriedmannRobertson-Walker background, each single polarization

\footnotetext{
${ }^{2}$ Since $h_{i j}$ is transverse and traceless, the direction of propagation can be chosen to lie along the third axis and, in this case the two physical polarizations of the graviton will be $h_{1}^{1}=$ $-h_{2}^{2}=h_{\oplus}$ and $h_{1}^{2}=h_{2}^{1}=h_{\otimes}$. This will be the nomenclature followed in the present paper.

${ }^{3}$ In the present notations the Planck length will be defined as $\ell_{\mathrm{P}}=\sqrt{8 \pi G}$ in units $\hbar=c=1$. Natural gravitational units $16 \pi G=2 \ell_{\mathrm{p}}^{2}=1$ will also be adopted when needed.

${ }^{4}$ Recently in [37] the field theoretical formulation of General Relativity was further developed with the purpose of deriving an energy-momentum tensor for the gravitational field. Our approach, in the present context, is more modest since we simply want to compare the dynamical consequences of different possible definitions of the energy-momentum pseudotensor of the relic gravitons.
}

of the graviton obeys, up to total derivatives, the action of a minimally coupled scalar degree of freedom

$$
\delta_{\mathrm{t}}^{(2)} S=\frac{1}{2} \int d^{4} x a^{2}(\tau) \eta^{\alpha \beta} \partial_{\alpha} h \partial_{\beta} h,
$$

where the dimensionless amplitude $h$ has been defined as

$$
h=\frac{h_{\oplus}}{\sqrt{2} \ell_{\mathrm{P}}}=\frac{h_{\circledast}}{\sqrt{2} \ell_{\mathrm{P}}} .
$$

Consequently, following [38], a natural ansatz for describing the backreaction of the created gravitons on the background space-time would be to use, for each single polarization, the energy-momentum tensor of a minimally coupled scalar field. This approach has been exploited, for instance, in [6] and in [17] for estimating the energy and pressure densities of the relic gravitons in a multistage Universe. A possible weakness of this second approach is that Eq. (3.4) holds, strictly speaking, in a FriedmannRoberston-Walker background. In spite of this, it is clearly useful to bear in mind this possibility.

The purpose of the present paper is to scrutinize the dynamical backreaction effects of the relic gravitons in the extreme situation where the background geometry undergoes a contraction that smoothly evolves, through a bounce, into an expanding phase. This sequence of events takes place, for instance, in pre-big bang models. The bouncing behavior is related to strong deviations from a radiative Universe. Consequently, short wave fluctuations are expected to modify or even restrict the dynamical range of bouncing models since, in this regime, the effective equation of state of relic gravitons is the one of radiation. For long wavelength fluctuations, the qualitative expectation is more difficult to formulate since, as already mentioned, the effective equation of state is sensitive to the specific features of the background evolution before and after the bounce.

Consider then, as an example, one of the simplest realizations of bouncing dynamics, i.e. the one provided by minimal pre-big bang models where, in the Einstein frame, the Universe first contracts as $a(\tau) \sim \sqrt{-\tau / \tau_{1}}$ and then expands, in the post-big bang phase, as $a(\tau) \sim \sqrt{\tau / \tau_{1}}$. An accurate description of the bouncing regime in the region $-\tau_{1}<\tau<\tau_{1}$ may be achieved, for instance, by means of a nonlocal potentials [40-42] that depend on the so-called shifted dilaton which is usually introduced in the context of the scale-factor-duality symmetry [10] (or more generally, in the treatment of $O(d, d)$ transformations [43,44] acting on the background fields of the low-energy string effective action). The features of the regular bouncing solutions discussed in $[45,46]$ are such that the rôle of the dilaton potential is only crucial in the bouncing region while, away from the bounce (i.e. $|\tau|>\tau_{1}$ ) the geometry is driven by the kinetic energy of the dilaton field.

As soon as the tensor modes reach into the superadiabatic regime, i.e. $k \tau \ll 1$ the effective pressure density of 
the relic gravitons becomes $\left\langle p_{\mathrm{gw}}\right\rangle \simeq\left\langle\rho_{\mathrm{gw}}\right\rangle$. Consequently, at least up to the bounce, the energy density of the relic gravitons is not likely dominate on the energy density of the dilatonic sources since the two components evolve exactly in the same way with the scale factor. While it is plausible that only around the bounce the short wavelength modes become dynamically relevant, it is also clear that the whole qualitative picture should be corroborated by a detailed numerical and analytical treatment.

It will be instructive to conduct the calculation of the dynamical backreaction within different ansatz for the energy-momentum pseudotensor. Then the results will be compared. A by-product of the present analysis will indeed be that different forms of the energy-momentum pseudotensor lead, quantitatively, to the same backreaction effects.

A final remark concerns the use of the Einstein frame where all the calculations will be presented. In fact, the Einstein frame is particularly useful for the comparison of the obtained results arising in different cosmological situations where the dilaton field is totally absent (such as the case of the transition from a de Sitter stage of expansion to a radiation-dominated phase). At the same time, the calculations could be conducted in the string (or Jordan) frame. In the string frame the dilaton field enters the action coupled to the Einstein-Hilbert term. In the Einstein frame the action is diagonal in the sense that the dilaton does not mix with the Ricci scalar. The Einstein and string metric tensors (and the scale factors) are related, in four dimensions, as

$$
G_{\mu \nu}^{\mathrm{s}}=e^{\varphi} G_{\mu \nu}^{\mathrm{E}}, \quad a_{\mathrm{s}}=a_{\mathrm{E}} e^{\varphi / 2} .
$$

From Eq. (1.6), bearing in mind the conventions of Eq. (1.1), the tensor modes are defined, in each of the two frames, as

$$
\delta_{\mathrm{t}} g_{i j}^{\mathrm{E}}=-a_{\mathrm{E}}^{2} h_{i j}, \quad \delta_{\mathrm{t}} G_{i j}^{\mathrm{s}}=-a_{\mathrm{s}}^{2} \tilde{h}_{i j},
$$

where both $h_{i j}$ and $\tilde{h}_{i j}$ are traceless and divergenceless. Now, from the first of the two equations reported in (1.6), we also have that

$$
\delta_{\mathrm{t}} G_{i j}^{\mathrm{s}}=e^{\varphi} \delta_{\mathrm{t}} g_{i j}^{\mathrm{E}} .
$$

This last equality holds in the case of tensor modes. In the case of scalar modes its form changes because the fluctuation of the dilaton field contributes to the scalar modes. Therefore, inserting Eq. (1.7) into Eq. (1.8) and recalling the relations between the scale factor in the two frames [second relation in Eq. (1.6)], we have that $h_{i j}=\tilde{h}_{i j}$, so the amplitude of the tensor modes are the same in both frames. Therefore, there is no surprise that if one computes $\tilde{h}_{i j}$ (in the string frame, i.e. using as background the appropriate $a_{\mathrm{s}}$ ) one finds exactly the same $h_{i j}$ obtainable in the Einstein frame (where the scale factor is $a_{\mathrm{E}}$ ). Of course the canonical action for the single tensor polarization in the string frame is (formally) different from the one reported in Eq. (1.4),

$$
\delta_{\mathrm{t}}^{(2)} \tilde{S}=\frac{1}{2} \int d^{4} x a_{\mathrm{s}}^{2}(\tau) e^{-\varphi} \eta^{\alpha \beta} \partial_{\alpha} \tilde{h} \partial_{\beta} \tilde{h} .
$$

Again, using Eq. (1.6) and recalling, as previously shown, that $\tilde{h}=h$, Eqs. (1.4) and (1.9) coincide. Notice, in fact, that by going from the String to the Einstein frame (or vice versa) the conformal time coordinate $\tau$ is invariant. From Eq. (1.9) one can read-off the correct normal modes to be quantized in the string frame. We checked explicitly that various "observables" (such as the mixing coefficients) have exactly the same numerical dependence upon the comoving momentum if they are evaluated either in the string frame or in the Einstein frame.

The present paper is then organized as follows. In Sec. II a class of string inspired bouncing cosmologies will be introduced. Section III is devoted to the analysis of the production of relic gravitons and to the different definitions of their energy-momentum pseudotensor in the framework of bouncing solutions. Section IV deals with the analytical estimates of the effective barotropic indices. Section V describes the implementation of a self-consistent (iterative) scheme that allows to compute the backreaction effects of relic gravitons. Section VI summarizes the main lessons to be drawn from the present analysis and contains also the concluding remarks. Finally, various technical results that are relevant for the present investigation are reported in the appendix.

\section{A CLASS OF REGULAR CURVATURE BOUNCES}

Regular bouncing solutions can be obtained by means of dilaton potentials that respect the scale-factor duality symmetry [10]. These potentials do not depend solely upon the dilaton field $\phi$ but rather upon the so-called shifted dilaton, i.e. $\bar{\phi}=\phi-\log \sqrt{-G_{\mathrm{s}}}\left[40-42\right.$ ] (see also [47]) where $G_{\mathrm{s}}$ is the determinant of the four-dimensional metric in the string frame and in the cosmic time coordinate.

Therefore the starting point of the present analysis is a generally covariant action supplemented by a nonlocal dilaton potential $[45,46,48]$. These equations are reported in appendix A. In particular, Eqs. (A3) and (A4) will now be written for the case of a four-dimensional background geometry characterized by a conformally flat FriedmannRobertson-Walker metric and by a homogeneous dilaton field: ${ }^{5}$

$$
g_{\mu \nu}=a^{2}(\tau) \eta_{\mu \nu}, \quad \phi=\phi(\tau) .
$$

Using Eq. (2.1), the set of homogeneous equations stemming from Eqs. (A3) and (A4) can be linearly combined and the relevant set of evolution equations, in units

\footnotetext{
${ }^{5}$ In the following all the quantities will be defined in the Einstein frame so that no ambiguity will arise in dropping the subscripts referring to the specific frame.
} 
$2 \ell_{\mathrm{P}}^{2}=1$, becomes

$$
\begin{gathered}
\mathcal{H}^{2}=\frac{\phi^{\prime 2}}{12}+\frac{e^{\phi} a^{2}}{6} V, \\
\mathcal{H}^{\prime}=-\frac{\phi^{\prime 2}}{6}+\frac{a^{2} e^{\phi}}{6}\left[V-\frac{3}{2} \frac{\partial V}{\partial \bar{\phi}}\right], \\
\phi^{\prime \prime}+2 \mathcal{H} \phi^{\prime}=-e^{\phi} a^{2}\left[V-\frac{1}{2} \frac{\partial V}{\partial \bar{\phi}}\right],
\end{gathered}
$$

where the prime will denote, from now on, a derivation with respect to the conformal time coordinate $\tau$ and $\mathcal{H}=a^{\prime} / a$.

By summing $\mathcal{H}^{\prime}$ [from Eq. (2.3)] to $2 \mathcal{H}^{2}$ [from Eq. (2.2)], the right-hand side of the obtained equation reconstructs, up to a numerical coefficient, the right-hand side of Eq. (2.4) so that the dependence on the potential terms may be eliminated. The remaining equation becomes then particularly simple:

$$
\frac{d}{d \tau}\left[a^{2}\left(\mathcal{H}+\frac{\phi^{\prime}}{2}\right)\right]=0 .
$$

Direct integration of Eq. (2.5) gives then:

$$
\mathcal{H}+\frac{\phi^{\prime}}{2}=\frac{\epsilon}{a^{2}},
$$

where $\epsilon$ is the integration constant. Unfortunately, in the conformal time coordinate the integration cannot proceed further with analytical methods. However, Eq. (2.6) can indeed be integrated analytically by defining the new time coordinate $\sigma$, i.e.

$$
d \sigma=\frac{d \tau}{a^{2}(\tau)} .
$$

Since the relation of $\tau$ to cosmic time is $d t=a(\tau) d \tau$, the relation of the cosmic time $t$ to the $\sigma$-parametrization is simply $d t=a^{3}(\sigma) d \sigma$. Equation (2.7) can then be used into Eq. (2.6) and the result is

$$
\mathcal{F}+\frac{d \phi}{d \sigma}=\epsilon,
$$

where $\mathcal{F}=(\ln a), \sigma$ and its relation to $\mathcal{H}$ is simply $\mathcal{H}=$ $\mathcal{F} / a^{2}$. By integrating once Eq. (2.8) and by naming as $\sigma_{1}$ the new integration constant the following explicit relation between the scale factor and the dilaton field can be obtained

$$
a(\sigma) e^{\phi(\sigma) / 2}=e^{\epsilon\left(\sigma+\sigma_{1}\right)} .
$$

Equation (2.9) simply fixes a relationship between $a(\sigma)$ and $\phi(\sigma)$; such a relationship must hold for any potential. Conversely, the particular form of the dilaton potential plays a crucial role in determining the relative evolution of the scale factor and of the dilaton whose combination appears directly in Eq. (2.9). Finally, once the potential is chosen, the Hamiltonian constraint of Eq. (2.2) selects the specific value of $\epsilon$.
To proceed even further it is necessary to write Eqs. (2.2), (2.3), and (2.4) in the parametrization defined by Eq. (2.7); in doing so it is useful to notice that

$$
\mathcal{H} \rightarrow \frac{\mathcal{F}}{a^{2}}, \quad \mathcal{H}^{\prime} \rightarrow \frac{1}{a^{2}}\left(\frac{d \mathcal{F}}{d \sigma}-2 \mathcal{F}^{2}\right) .
$$

Consequently, Eqs. (2.2), (2.3), and (2.4) can be written as

$$
\begin{gathered}
\mathcal{F}^{2}=\frac{\xi^{2}}{12}+\frac{e^{\phi} a^{6}}{6} V . \\
\frac{d \mathcal{F}}{d \sigma}=\frac{e^{\phi} a^{6}}{2}\left[V-\frac{1}{2} \frac{\partial V}{\partial \bar{\phi}}\right], \\
\frac{d \xi}{d \sigma}=-e^{\phi} a^{6}\left[V-\frac{1}{2} \frac{\partial V}{\partial \bar{\phi}}\right],
\end{gathered}
$$

where $\xi=d \phi / d \sigma$. Equations (2.11) and (2.13) stem directly from Eq. (2.2) and (2.4) by using Eqs. (2.10). Equation (2.12) follows from Eq. (2.3) by eliminating, in the obtained expression, the dependence on $\mathcal{F}^{2}$ through Eq. (2.11).

The solutions of the system formed by Eqs. (2.11), (2.12), and (2.13) can now be derived for the general class of potentials $V=-V_{0} e^{\alpha \bar{\phi}}$. In this case we have

$$
\begin{aligned}
& a^{6} e^{\phi} V=-V_{0} a^{6} e^{\phi+\alpha \bar{\phi}}=-V_{0} \frac{e^{-(\alpha-2) \phi / 2}}{a^{3(\alpha-2)}}, \\
& a^{6} e^{\phi}\left[V-\frac{1}{2} \frac{\partial V}{\partial \bar{\phi}}\right]=\frac{\alpha-2}{2} V_{0} \frac{e^{-(\alpha-2) \phi / 2}}{a^{3(\alpha-2)}},
\end{aligned}
$$

where we used that $\bar{\phi}=\phi-3 \ln a_{\mathrm{s}}, a_{\mathrm{s}}(\tau)$ being the scale factor in the string frame metric. Furthermore, bearing in mind that the scale factor in the Einstein frame is given by $a=e^{\phi / 2} a_{\mathrm{s}}$, Eqs. (2.14) follow immediately from the definition of the potential in terms of $\bar{\phi}$.

Equations (2.11) and (2.12) can now be combined and the result is

$$
\frac{d \mathcal{F}}{d \sigma}+\frac{3}{2}(\alpha-2) \mathcal{F}^{2}=\frac{\alpha-2}{8} \xi^{2} .
$$

On the other hand, Eq. (2.6) allows to express $\xi$ in terms of $\epsilon$ and $\mathcal{F}$, i.e.

$$
\frac{\xi}{2}=\epsilon-\mathcal{F} .
$$

Eliminating $\xi$ from Eq. (2.15) through Eq. (2.16) the whole problem can be reduced to the following equation

$$
\frac{d \mathcal{F}}{d \sigma}=\frac{\alpha-2}{2}\left[\epsilon^{2}-2 \mathcal{F}^{2}-2 \epsilon \mathcal{F}\right],
$$

that can be easily integrated with the result that $\mathcal{F}$ is

$$
\mathcal{F}=-\frac{\epsilon}{2}+\frac{\sqrt{3} \epsilon}{2} \tanh \left[\frac{\beta\left(\sigma+\sigma_{2}\right)}{2}\right],
$$



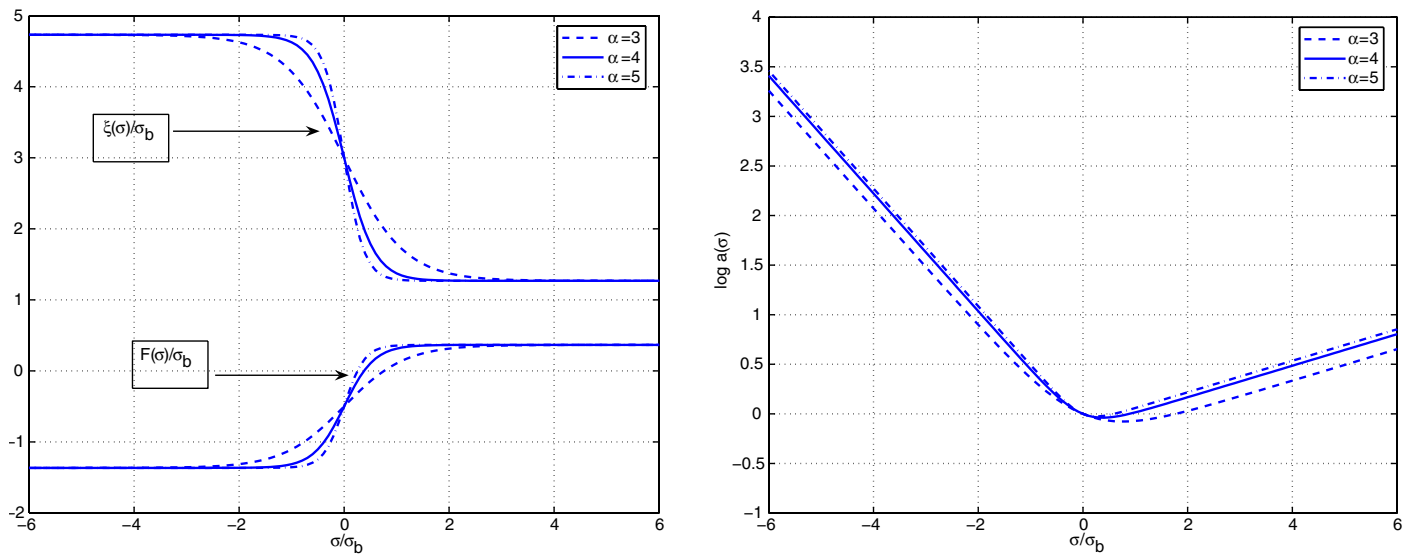

FIG. 1 (color online). In the left plot the evolution of $\mathcal{F} / \sigma_{\mathrm{b}}$ and $\xi / \sigma_{\mathrm{b}}$ are illustrated for different values of the parameter $\alpha$. In the right plot the logarithm (to base 10) of the scale factor is illustrated for different values of $\alpha$.

where $\beta=\sqrt{3} \epsilon(\alpha-2)$ and $\sigma_{2}$ is an integration constant. Inserting Eq. (2.18) into Eq. (2.16), the explicit form of $\xi$ turns out to be

$$
\xi=3 \epsilon-\sqrt{3} \epsilon \tanh \left[\frac{\beta\left(\sigma+\sigma_{2}\right)}{2}\right] .
$$

Equations (2.18) and (2.19) can be integrated again so that the explicit functional dependence of the dilaton and of the scale factor upon the time coordinate $\sigma$ becomes:

$$
\begin{aligned}
& a(\sigma)=e^{-\phi_{\mathrm{b}} / 2} e^{-\left(\epsilon\left(\sigma-2 \sigma_{1}\right) / 2\right)}\left[\cosh \frac{\beta\left(\sigma+\sigma_{2}\right)}{2}\right]^{1 / \alpha-2}, \\
& \phi(\sigma)=\phi_{\mathrm{b}}+3 \epsilon \sigma-\frac{2}{\alpha-2} \ln \cosh \frac{\beta\left(\sigma+\sigma_{2}\right)}{2} .
\end{aligned}
$$

The consistency of Eqs. (2.20) and (2.21) with the Hamiltonian constraint (2.11) demands that the value of $\epsilon$ is fixed as:

$$
3 \epsilon^{2}=V_{0} e^{(\alpha-2) \phi_{\mathrm{b}}} e^{-3(\alpha-2) \sigma_{1}}
$$

Equation (2.20) can now be inserted into Eq. (2.7) to obtain the asymptotic relation (i.e. for $\sigma \rightarrow \pm \infty$ ) between the $\sigma$ and $\tau$. This relation is logarithmic in the sense that, up to numerical factors $\sigma \simeq-\ln (-\tau)$ (for $\sigma$ and $\tau$ going to $-\infty$ ) and $\sigma \simeq \ln \tau$ (for $\sigma$ and $\tau$ going to $+\infty$ ). This is particularly appropriate for numerical purposes since, in practice, the $\sigma$-parametrization acts as a logarithmic scale in the asymptotic regions. On the contrary, the central region of the dynamics (i.e. $\sigma \simeq \sigma_{2}$ ) is described by a linear time-scale.

To describe the features of the obtained solution let us fix, for simplicity, $\sigma_{1}=\sigma_{2}=0$. In short the basic feature of the solution are the following:

(a) for $\alpha>2$ the solution (2.20) and (2.21) describes a smooth interpolation between a contracting regime (i.e. $\mathcal{F}<0$ ) for $\sigma<0$ and an expanding solution, i.e. $\mathcal{F}>0$ valid for $\sigma>0$; (b) for $\alpha<2$ the dynamical sequence is reversed since the scale factor expands for $\sigma<0$ and contracts for $\sigma>0$, as is can be argued from the specific form of $\mathcal{F}$ (see Eq. (2.18));

(c) for $\alpha=2$ the solution always contract and the bouncing behavior is not realized.

If $\sigma_{1}$ or $\sigma_{2}$ (or both) are different from zero the solutions are not centered around $\sigma=0$ and the same discussion applies for an appropriately translated coordinate system.

Concerning the third item of the above list, it is interesting to notice that, in the case $\alpha=2$, the explicit solution reads

$$
\begin{gathered}
a(\sigma)=e^{-\phi_{\mathrm{b}} / 2} e^{-\epsilon \sigma / 2}, \quad \phi(\sigma)=3 \epsilon \sigma+\phi_{\mathrm{b}}, \\
3 \epsilon^{2}=V_{0},
\end{gathered}
$$

implying that $\mathcal{F}=-\epsilon / 2$. In Fig. 1 (left panel) the evolution of $\xi(\sigma)$ and $\mathcal{F}(\sigma)$ is illustrated as it emerges from Eqs. (2.18) and (2.19). The time scale is given in terms of $\sigma_{\mathrm{b}}=1 / \epsilon$, i.e. the typical bouncing time. In the left plot, the logarithm ${ }^{6}$ (to base 10) of the scale factor is illustrated for the same range of $\alpha$. The initial conditions for the solutions can be characterized in terms of $\phi$ or more precisely $e^{\phi / 2}$ that measures the initial strength of the gauge coupling. We shall be primarily interested in the case when the solution contracts for $\sigma<0$ and expands for $\sigma>0$ (i.e. $\alpha>2$ ). In this situation the pre-big bang initial conditions will be reproduced since $e^{\phi / 2} \ll 1$ for $\sigma \rightarrow$ $-\infty$. Notice that $\phi_{\mathrm{b}}$ measures the strength of the gauge coupling around the bounce, i.e. for $\sigma \simeq 0$. Of particular interest will be the case when $\phi_{\mathrm{b}} \simeq 0$, i.e. when strong coupling is reached just at the bounce. It is clearly always possible to tune $\phi_{\mathrm{b}}$ in such a way that the gauge coupling is

\footnotetext{
${ }^{6}$ In the present paper the natural logarithm will always be denoted as ln while the logarithm to base 10 will be denoted as $\log$.
} 
much smaller than 1 but we shall try to avoid this tuning and examine the more realistic situation where $\phi_{\mathrm{b}} \simeq 0$.

The $\sigma$-parametrization is particularly useful for the numerical evolution of the fluctuations and for the selfconsistent treatment of the dynamical backreaction effects. It is sometimes practical, in a complementary perspective, to solve for the mode functions directly in the conformal time parametrization. As already remarked, in the conformal time parametrization we cannot rely on exact solutions. Therefore the idea will be to map the asymptotic solutions in the $\sigma$ parametrization into asymptotic solutions in the $\tau$ parametrization and then solve numerically Eqs. (2.2), (2.3), and (2.4). It is therefore necessary to develop the numerical intuition for the evolution of the background in the conformal time parametrization. Of particular technical relevance is the precise mapping of the initial conditions from the $\sigma$-parametrization to the conformal time coordinate.

To fix the ideas, consider the case $\alpha=4$ with the bounce occurs around $\sigma_{1}=\sigma_{2}=0$. In this case the mapping of the initial conditions between the $\sigma$ and the $\tau$ parametrization can be derived from the following chain of relations

$$
3 \epsilon^{2}=V_{0} e^{2 \phi_{\mathrm{b}}}=\frac{e^{-2 \phi_{\mathrm{b}}}}{\tau_{1}^{2}},
$$

where the first equality is nothing but Eq. (2.22) in the case $\alpha=4$ (and $\sigma_{1}=0$ ). The second equality is fixed by solving directly the Hamiltonian constraint of Eq. (2.2) in the asymptotic regions (i.e. $\tau \rightarrow \pm \infty$ ). Equation (2.24) fixes the relation between the bouncing time in the $\sigma$-parametrization, i.e. $\sigma_{\mathrm{b}}=1 / \epsilon$, in terms of the bouncing time in the $\tau$-parametrization, i.e. $\tau_{1}$. In Fig. 2 the numerical integration of Eqs. (2.2), (2.3), and (2.4) is illustrated in terms of the scale factor. In particular, for $\tau<-\tau_{1}$ the solution is well approximated by

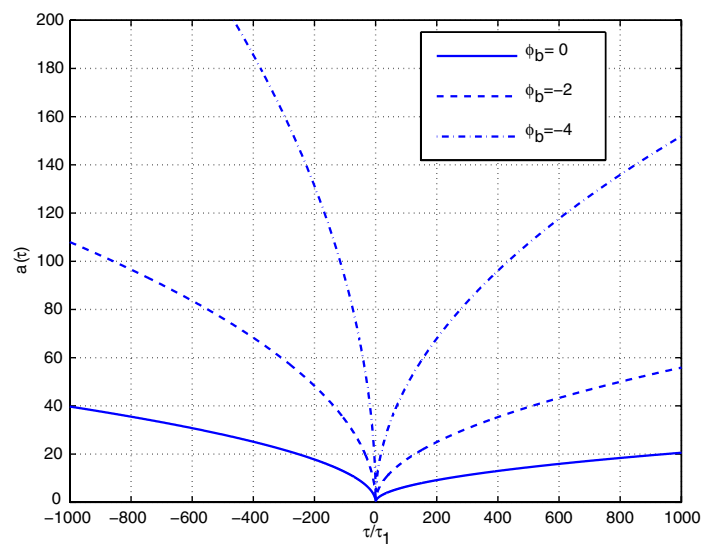

$$
a(\tau)=a_{-} \sqrt{\frac{-\tau}{2 \tau_{1}}}, \quad a_{-}=e^{-\phi_{\mathrm{b}} / 2} \sqrt{\frac{2(\sqrt{3}+1)}{\sqrt{3}}},
$$

while for $\tau>-\tau_{1}$ the solution is well approximated by

$$
a(\tau)=a_{+} \sqrt{\frac{\tau}{2 \tau_{1}}}, \quad a_{+}=e^{-\phi_{\mathrm{b}} / 2} \sqrt{\frac{2(\sqrt{3}-1)}{\sqrt{3}}},
$$

where $\phi_{\mathrm{b}}$ is the same constant introduced in Eq. (2.21). In the prebounce region the evolution of the dilaton can be obtained from Eq. (2.21) by using the asymptotic relation between $\tau$ and $\sigma$ in the limit $\tau \rightarrow-\infty$ and $\sigma \rightarrow-\infty$. The result is

$$
\begin{aligned}
\phi(\tau)= & \phi_{\mathrm{b}}-(\sqrt{3}-1) \ln 2-\sqrt{3} \ln \left(\frac{\sqrt{3}+1}{\sqrt{3}}\right) \\
& -\sqrt{3} \ln \left(-\frac{\tau}{\tau_{1}}\right) .
\end{aligned}
$$

In the intermediate region, the minimum of the scale factor corresponds to

$$
a_{\min }=0.61 e^{-\phi_{\mathrm{b}} / 2} .
$$

The "width" of the bounce is illustrated in Fig. 2 (right plot) and can be estimated analytically as

$$
\frac{\tau_{-}}{\tau_{1}}=-\frac{0.74}{a_{-}^{2}} e^{-\phi_{\mathrm{b}}}, \quad \frac{\tau_{+}}{\tau_{1}}=\frac{0.74}{a_{+}^{2}} e^{-\phi_{\mathrm{b}}} .
$$

In Fig. 2 the values of $\tau_{ \pm}$correspond to the two vertical arrows around the origin.

By changing the various parameters it appears that both $a^{2} H^{2}$ and $\phi^{\prime 2} / 2$ are not sensitive to the value of $\phi_{\mathrm{b}}$ but rather they depend on $\tau_{1}$. In particular, it turns out that

$$
\mathcal{H}^{2} \simeq \frac{0.61}{\tau_{1}^{2}}, \quad \frac{\phi^{\prime 2}}{2} \simeq \frac{7.5}{\tau_{1}^{2}} .
$$

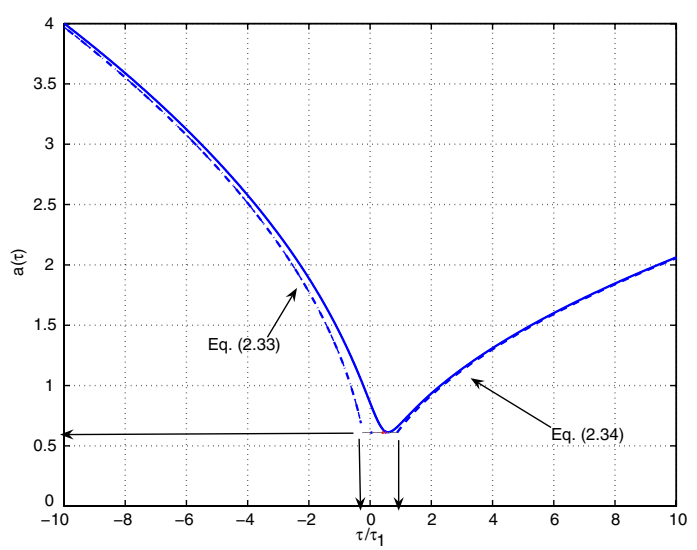

FIG. 2 (color online). The evolution of the scale factor (left panel) is illustrated for different initial values of the gauge coupling. In the right plot the analytical approximations for the evolution of the scale factor (dashed lines) are compared with the numerical solution. 
In the case $\alpha=2$ the solution can be expressed exactly also in the conformal time parametrization. Recalling Eq. (2.7) we obtain that

$$
\begin{gathered}
\sigma=-\frac{1}{\epsilon} \ln (-\epsilon) \tau, \quad a(\tau)=e^{-\phi_{\mathrm{b}} / 2} \sqrt{-\epsilon \tau}, \\
\phi(\tau)=\phi_{\mathrm{b}}-3 \ln (-\epsilon \tau) .
\end{gathered}
$$

Thus, unlike the case $\alpha>3$ the solution with $\alpha=2$ is singular in the conformal time parametrization.

The $\sigma$ parametrization has one more important feature that has been already implicitly treated in Eq. (2.10). While the sign of $\mathcal{H}$ and $\mathcal{F}$ is preserved by the mapping $\tau \rightarrow \sigma$, the sign of $\mathcal{H}^{\prime}$ is, in general, different from the sign of $\mathcal{F}$, $\sigma$. The simplest way of realizing this aspect is to notice that, from Eqs. (2.7) and (2.10) we can write

$$
\mathcal{F}=a^{2} \mathcal{H}, \quad \frac{\partial \mathcal{F}}{\partial \sigma}=a^{4}\left(\mathcal{H}^{\prime}+2 \mathcal{H}^{2}\right) .
$$

Consider now the simple case of a radiation-dominated Universe. In this case $a(\tau)=\left(\tau+2 \tau_{1}\right) / \tau_{1}$ for $\tau \geq-\tau_{1}$. Consequently, from Eq. (2.32), $\mathcal{H}$ and $\mathcal{F}$ will have the same sign but $\mathcal{F}_{, \sigma}$ will be always positive while $\mathcal{H}^{\prime}=$ $-1 /\left(\tau+2 \tau_{1}\right)^{2}$ will always be negative. The cure for this type of problem is very simple. The invariant quantities (like for instance) the Ricci scalar are always the same in both coordinate systems. So, for instance, in a radiationdominated Universe the background Ricci scalar, i.e. $\bar{R}$ is always zero both in the $\tau$ and in the $\sigma$ parametrizations. In fact,

$$
\bar{R}=-\frac{6}{a^{2}}\left(\mathcal{H}^{2}+\mathcal{H}^{\prime}\right)=-\frac{6}{a^{6}}\left(\frac{\partial \mathcal{F}}{\partial \sigma}-\mathcal{F}^{2}\right),
$$

where the second equality follows by using Eq. (2.10). Now, it is easy to show that, for a radiation-dominated Universe, the evolution equation obeyed by $\mathcal{F}$ is exactly $\mathcal{F}_{, \sigma}=\mathcal{F}^{2}$.

It is appropriate to remark at this point that the bouncing solutions obtained here in the framework of the low-energy string effective action differ from the ones derived in the presence of spatial curvature and in a general relativistic context. For instance in $[49,50]$ (see also $[51,52]$ and references therein) spatially closed models driven by a massive scalar were studied. As anticipated, in this framework the dynamical sequence of events differs from the one discussed here. In $[49,50]$ the Universe first deflates, then bounces and finally inflates. ${ }^{7}$ In spite of this statement, the treatment the dynamical backreaction of relic gravitons in closed bounces can be developed by following the same methods discussed here.

\footnotetext{
${ }^{7}$ This class of closed bounces has a long history. For instance in $[53,54]$ useful analytical solutions were derived and exploited. In connection with this problem see also [55-57].
}

\section{ENERGY-MOMENTUM PSEUDOTENSOR(S)}

\section{A. General considerations}

Recalling Eq. (1.1), the quadratic action for $h_{i j}$ can be obtained by perturbing Eq. (A1) to second-order in the amplitude of the tensor fluctuations of the metric. Some relevant expressions can be found in the appendix B. Up to total derivatives, the result can be written as

$$
\delta_{\mathrm{t}}^{(2)} S=S_{\mathrm{gw}}=\frac{1}{8 \ell_{\mathrm{P}}^{2}} \int d^{3} x d \tau \sqrt{-\bar{g}} \partial_{\alpha} h_{i}^{j} \partial_{\beta} h_{j}^{i} \bar{g}^{\alpha \beta},
$$

where $\bar{g}_{\alpha \beta}=a^{2}(\tau) \eta_{\alpha \beta}$ and $\eta_{\alpha \beta}$ is the Minkowski metric.

As discussed in connection with Eq. (1.1), the two polarizations of the graviton can be chosen to be $h_{1}^{1}=$ $-h_{2}^{2}=h_{\oplus}$ and $h_{1}^{2}=h_{2}^{1}=h_{\otimes}$. Furthermore recalling the notations of Eq. (1.5) and (3.1) becomes, for a single tensor polarization

$$
S_{\mathrm{gw}}=\frac{1}{2} \int d^{3} x d \tau \sqrt{-\bar{g}} \partial_{\alpha} h \partial_{\beta} h \bar{g}^{\alpha \beta},
$$

Using the parametrization defined in Eq. (2.7) and (3.2) can be also written as

$$
S_{\mathrm{gw}}=\frac{1}{2} \int d^{3} x d \sigma\left[\left(\frac{\partial h}{\partial \sigma}\right)^{2}-a^{4}(\sigma) \partial_{m} h \partial^{m} h\right] .
$$

The associated canonical momentum, i.e. $\Pi=\partial_{\sigma} h$ allows one to obtain the Hamiltonian of the tensor modes, i.e.

$$
H_{\mathrm{gw}}(\sigma)=\frac{1}{2} \int d^{3} x\left[\Pi^{2}+a^{4} \partial_{m} h \partial^{m} h\right] .
$$

By promoting the classical fields to quantum mechanical operators we have that ${ }^{8}$

$$
\begin{aligned}
\hat{h}(\vec{x}, \sigma) & =\frac{1}{2(2 \pi)^{3 / 2}} \int d^{3} k\left[\hat{h}_{\vec{k}}(\sigma) e^{-i \vec{k} \cdot \vec{x}}+\hat{h}_{\vec{k}}^{\dagger}(\sigma) e^{i \vec{k} \cdot \vec{x}}\right], \\
\hat{\Pi}(\vec{x}, \sigma) & =\frac{1}{2(2 \pi)^{3 / 2}} \int d^{3} k\left[\hat{\Pi}_{\vec{k}}(\sigma) e^{-i \vec{k} \cdot \vec{x}}+\hat{\Pi}_{\vec{k}}^{\dagger}(\sigma) e^{i \vec{k} \cdot \vec{x}}\right] .
\end{aligned}
$$

In the Heisenberg representation the evolution of the field operators can be written as

$$
\begin{aligned}
& \hat{h}_{\vec{k}}(\sigma)=\hat{a}_{\vec{k}}\left(\sigma_{\mathrm{i}}\right) F_{k}(\sigma)+\hat{a}_{-\vec{k}}^{\dagger}\left(\sigma_{\mathrm{i}}\right) F_{k}(\sigma)^{*}, \\
& \hat{\Pi}_{\vec{k}}(\sigma)=\hat{a}_{\vec{k}}\left(\sigma_{\mathrm{i}}\right) G_{\vec{k}}(\sigma)+\hat{a}_{-\vec{k}}^{\dagger}\left(\sigma_{\mathrm{i}}\right) G_{k}(\sigma)^{*},
\end{aligned}
$$

where $\hat{a}_{\vec{k}}\left(\sigma_{\mathrm{i}}\right)$ and $\hat{a}_{-\vec{k}}\left(\sigma_{\mathrm{i}}\right)$ annihilate the vacuum for $\sigma_{\mathrm{i}} \rightarrow$ $-\infty$. The mode functions $F_{k}$ and $G_{k}$ now obey

$$
\frac{d^{2} F_{k}}{d \sigma^{2}}+\Omega^{2} F_{k}=0, \quad G_{k}=\frac{d F_{k}}{d \sigma},
$$

where $\Omega=k a^{2}(\sigma)$.

\footnotetext{
${ }^{8}$ Notice that, within our set of conventions, $\hat{h}_{-\vec{k}}^{\dagger}=\hat{h}_{\vec{k}}$ and $\hat{\Pi}_{-\vec{k}}^{\dagger}=\hat{\Pi}_{\vec{k}}$.
} 


\section{B. Mixing coefficients}

In the short wavelength limit, i.e. $k|\tau|>1$ the mode function $F_{k}(\sigma)$ has an oscillating behavior. This regime characterizes the asymptotics of the evolution of the evolution of the mode functions. Initially, for $\sigma \rightarrow-\infty$ the approximate solution of Eq. (3.8) is simply given by

$$
\begin{aligned}
& F_{k}(\sigma)=\frac{1}{\sqrt{2 \Omega}} e^{-i \int \Omega d \sigma}, \\
& G_{k}(\sigma)=-\frac{1}{\sqrt{2 \Omega}}[\mathcal{F}+i \Omega] e^{-i \int \Omega d \sigma} .
\end{aligned}
$$

In the limit $\sigma \rightarrow+\infty$ the solution can be expressed by means of two mixing coefficients $c_{+}(k)$ and $c_{-}(k)$, i.e.

$$
\begin{aligned}
F_{k}(\sigma)= & \frac{1}{\sqrt{2 \Omega}}\left[c_{+}(k) e^{-i \int \Omega d \sigma}+c_{-}(k) e^{i \int \Omega d \sigma}\right], \\
G_{k}(\sigma)= & \frac{1}{\sqrt{2 \Omega}}\left[-(\mathcal{F}+i \Omega) c_{+}(k) e^{-i \int \Omega d \sigma}\right. \\
& \left.+(i \Omega-\mathcal{F}) c_{-}(k) e^{i \int \Omega d \sigma}\right] .
\end{aligned}
$$

In the long wavelength limit, i.e. $k|\tau|<1$ the approximate solution of Eq. (3.8) across the bounce is even simpler and it is given by

$$
F_{k}=A_{k}+B_{k} \sigma, \quad G_{k}=B_{k} .
$$

It is relevant to remark here that the condition $k|\tau| \simeq 1$ reads, in the $\sigma$-parametrization,

$$
\left|\int \Omega d \sigma\right| \simeq 1 \text {. }
$$

Because of the presence of the absolute values, there will be two distinct moments, in the dynamical evolution, where the condition $k|\tau|=1$ is verified. Before the bounce, i.e. for $\sigma<0$, for a given mode $k$ the condition $-k \tau(\sigma) \simeq 1$ will define the time of "exit" in the $\sigma$ parametrization. The notation $\tau(\sigma)$ simply means that the specific relation between $\tau$ and $\sigma$ has to be derived from Eq. (2.7) by using the appropriate expression of the scale factor. After the bounce, i.e. for $\sigma>0$, the condition $k \tau(\sigma) \simeq 1$ will define the time of "reentry" in the $\sigma$-parametrization.

Defining conventionally the typical time scale of the bounce as $\sigma_{\mathrm{b}}=1 / \epsilon$, consider the expression of the scale factor given in Eq. (2.20). In units of bouncing times the exit and reentry occur, approximately, for

$$
\begin{gathered}
\frac{\sigma_{\mathrm{ex}}}{\sigma_{\mathrm{b}}}=-\frac{1}{\sqrt{3}+1} \ln \left[\frac{\sqrt{3}+1}{\kappa} e^{\phi_{\mathrm{b}}} 2^{2 /(\alpha-2)}\right], \\
\frac{\sigma_{\mathrm{re}}}{\sigma_{\mathrm{b}}}=\frac{1}{\sqrt{3}-1} \ln \left[\frac{\sqrt{3}-1}{\kappa} e^{\phi_{\mathrm{b}}} 2^{2 /(\alpha-2)}\right],
\end{gathered}
$$

where we defined $\kappa=k \sigma_{\mathrm{b}}$, i.e. the wave number in units of inverse bounce time. Equations (3.13) and (3.14) can be easily derived from Eq. (3.12) by using the explicit expressions of $a(\sigma)$ given in Eq. (2.20) evaluated, respectively, in the limits $\sigma<-\sigma_{\mathrm{b}}$ and $\sigma>\sigma_{\mathrm{b}}$. Equations (3.13) and (3.14) are derived by fixing $\sigma_{1}=\sigma_{2}=0$ in Eq. (2.20) and by using Eq. (2.7). Moreover, because of the considerations elaborated in the previous section, the expressions given in Eqs. (3.13) and (3.14) hold for $\alpha>2$.

For modes $\kappa<1$, the three solutions given in Eqs. (3.9), (3.10), and (3.11) can be appropriately matched in such a way that the field operators are continuous function of $\sigma$. This procedure leads to the determination of the coefficients $A_{k}, B_{k}$

$$
\begin{aligned}
A_{k} & =\frac{1}{\sqrt{2 \Omega_{\mathrm{ex}}}} e^{-i k \tau_{\mathrm{ex}}}\left[1+\left(\mathcal{F}_{\mathrm{ex}}+i \Omega_{\mathrm{ex}}\right) \sigma_{\mathrm{ex}}\right], \\
B_{k} & =-\frac{1}{\sqrt{2 \Omega_{\mathrm{ex}}}} e^{-i k \tau_{\mathrm{ex}}}\left(\mathcal{F}_{\mathrm{ex}}+i \Omega_{\mathrm{ex}}\right)
\end{aligned}
$$

and, ultimately, of the mixing coefficients $c_{ \pm}(k)$ :

$$
\begin{aligned}
c_{-}(k)= & -\frac{i}{2 \sqrt{\Omega_{\mathrm{ex}} \Omega_{\mathrm{re}}}} e^{-i k\left(\tau_{\mathrm{re}}+\tau_{\mathrm{ex}}\right)}\left[\left(\mathcal{F}_{\mathrm{re}}-\mathcal{F}_{\mathrm{ex}}\right)\right. \\
& +i\left(\Omega_{\mathrm{re}}-\Omega_{\mathrm{ex}}\right)-\left(\mathcal{F}_{\mathrm{re}}+i \Omega_{\mathrm{re}}\right)\left(\mathcal{F}_{\mathrm{ex}}+i \Omega_{\mathrm{ex}}\right) \\
& \left.\times\left(\sigma_{\mathrm{re}}-\sigma_{\mathrm{ex}}\right)\right],
\end{aligned}
$$

$$
\begin{aligned}
c_{+}(k)= & -\frac{i}{2 \sqrt{\Omega_{\mathrm{ex}} \Omega_{\mathrm{re}}}} e^{-i k\left(\tau_{\mathrm{ex}}-\tau_{\mathrm{re}}\right)}\left[\left(\mathcal{F}_{\mathrm{ex}}-\mathcal{F}_{\mathrm{re}}\right)\right. \\
& +i\left(\Omega_{\mathrm{re}}+\Omega_{\mathrm{ex}}\right)+\left(\mathcal{F}_{\mathrm{re}}-i \Omega_{\mathrm{re}}\right)\left(\mathcal{F}_{\mathrm{ex}}+i \Omega_{\mathrm{ex}}\right) \\
& \left.\times\left(\sigma_{\mathrm{re}}-\sigma_{\mathrm{ex}}\right)\right] .
\end{aligned}
$$

In Eqs. (3.15), (3.16), and (3.17), the obvious notations $\Omega_{\mathrm{ex}}=\Omega\left(\sigma_{\mathrm{ex}}\right), \mathcal{F}_{\mathrm{ex}}=\mathcal{F}\left(\sigma_{\mathrm{ex}}\right)$ (and similarly for $\Omega_{\mathrm{re}}$ and $\mathcal{F}_{\text {re }}$ ) have been employed. Equations (3.16) and (3.17) imply that $\left|c_{+}(k)\right|^{2}-\left|c_{-}(k)\right|^{2}=1$ which is a simple consequence of the unitary evolution. For $\kappa>1$ the mixing coefficient is exponentially suppressed. To assess accurately the form of exponential suppression, a numerical treatment is necessary. The strategy here is to integrate numerically Eqs. (3.8) with initial conditions given by Eq. (3.9) and to obtain, numerically the mixing coefficients, i.e.

$$
\left|c_{+}(k)\right|^{2}-\left|c_{-}(k)\right|^{2}=i\left[F_{k}^{*}(\sigma) G_{k}(\sigma)-F_{k}(\sigma) G_{k}^{*}(\sigma)\right],
$$

$$
\begin{aligned}
\left|c_{+}(k)\right|^{2}+\left|c_{-}(k)\right|^{2}= & \frac{1}{\Omega}\left\{\left|G_{k}(\sigma)\right|^{2}+\left(\mathcal{F}^{2}+\Omega^{2}\right)\left|F_{k}(\sigma)\right|^{2}\right. \\
& \left.+\mathcal{F}\left[F_{k}^{*}(\sigma) G_{k}(\sigma)+F_{k}(\sigma) G_{k}^{*}(\sigma)\right]\right\} .
\end{aligned}
$$

For the numerical consistency of the whole approach, it must be true for each specific numerical integration, that the combination reported in Eq. (3.18), corresponding to the Wronskian of the solution, is equal to 1 [since it is 
chosen to be equal to one at the initial integration time, see Eq. (3.9)]. The numerical accuracy of the Wronskian normalization condition is a check of the consistency of the whole approach. Along the same lines, the combination in Eq. (3.19) must go to a constant for $\sigma \rightarrow+\infty$.

A set of first-order equations valid for the mixing coefficients will now be discussed. Defining

$$
e_{ \pm}=e^{ \pm i \int \Omega d \sigma},
$$

it is possible to find that the mixing coefficients obey

$$
\frac{d c_{+}}{d \sigma}=\frac{1}{2} \frac{d \ln \Omega}{d \sigma} e_{+}^{2} c_{-}, \quad \frac{d c_{-}}{d \sigma}=\frac{1}{2} \frac{d \ln \Omega}{d \sigma} e_{-}^{2} c_{+} .
$$

Equation (3.21) was derived, in a related context, in Ref. [16]. Since $c_{+}(k)$ and $c_{-}(k)$ are two complex quantities subjected to the condition $\left|c_{+}(k)\right|^{2}-\left|c_{-}(k)\right|^{2}=1$, they correspond, overall to three real variables that can be chosen to be

$$
\begin{aligned}
\bar{n}_{k} & =\left|c_{-}(k)\right|^{2}, \\
p_{k} & =c_{+}(k) c_{-}^{*}(k) e_{-}^{2}+c_{-}(k) c_{+}^{*}(k) e_{+}^{2}, \\
q_{k} & =i\left[c_{+}(k) c_{-}^{*}(k) e_{-}^{2}-c_{+}^{*}(k) c_{-}(k) e_{+}^{2}\right] .
\end{aligned}
$$

The quantities $\bar{n}_{k}, p_{k}$ and $q_{k}$ obey the following set of equations

$$
\begin{gathered}
\frac{d \bar{n}_{k}}{d \sigma}=\mathcal{F} p_{k}, \\
\frac{d p_{k}}{d \sigma}=2 \mathcal{F}\left(1+2 \bar{n}_{k}\right)-2 \Omega q_{k}, \\
\frac{d q_{k}}{d \sigma}=2 \Omega p_{k} .
\end{gathered}
$$

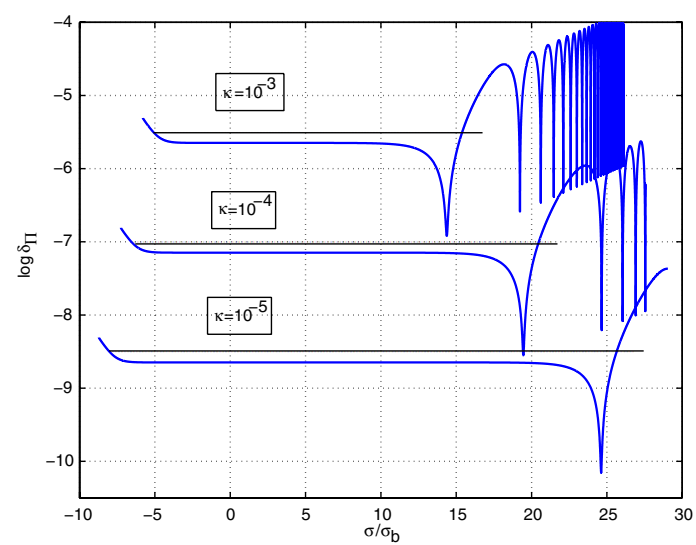

The initial conditions should be such that, initially, $c_{+}(k)=1$ and $c_{-}=0$, i.e.

$$
\bar{n}_{k}\left(\sigma_{\mathrm{i}}\right)=0, \quad p_{k}\left(\sigma_{\mathrm{i}}\right)=0, \quad q_{k}\left(\sigma_{\mathrm{i}}\right)=0 .
$$

Now the analytical estimates will be corroborated by numerical integration. One of the pleasant features of the $\sigma$-parametrization is that the spectrum of the canonical momenta is strictly constant in the long wavelength limit. The spectrum of canonical momenta is the Fourier transform of the two-point correlation function of $\hat{\Pi}(\vec{x}, \sigma)$, i.e.

$$
\langle\hat{\Pi}(\vec{x}, \sigma) \hat{\Pi}(\vec{y}, \sigma)\rangle=\int d \ln k\left|\delta_{\Pi}(k, \sigma)\right|^{2} \frac{\sin k r}{k r},
$$

where

$$
\left|\delta_{\Pi}(k, \sigma)\right|^{2}=\frac{k^{3}}{2 \pi^{2}}\left|G_{k}(\sigma)\right|^{2} .
$$

Equations (3.27) and (3.28) can be obtained by using Eqs. (3.5) together with Eqs. (3.6) and (3.7). When performing the appropriate quantum mechanical expectation values (in the Heisenberg representation) it should be recalled that $\left\langle\hat{a}_{\vec{k}} \hat{a}_{\vec{p}}^{\dagger}\right\rangle=\delta^{(3)}(\vec{k}-\vec{p})$.

Consequently, from Eq. (3.11) and from the second relation reported in Eq. (3.15) we get

$$
\delta_{\Pi}(k, \sigma)=\frac{k^{3 / 2}}{2 \pi} \sqrt{\frac{\mathcal{F}_{\mathrm{ex}}^{2}+\Omega_{\mathrm{ex}}^{2}}{\Omega_{\mathrm{ex}}}},
$$

where the quantities at the right hand side are fully determined analytically by using Eqs. (3.13) and (3.15) and by recalling Eq. (2.18). In Fig. 3 (left panel), the analytical expectation given by Eq. (3.29) is compared with the numerical result obtained by integrating Eqs. (3.8) with initial conditions dictated by Eq. (3.10). In Fig. 3 (plot at the right) the numerical result for the integration of Eqs. (3.8) with initial conditions dictated by Eq. (3.10) is

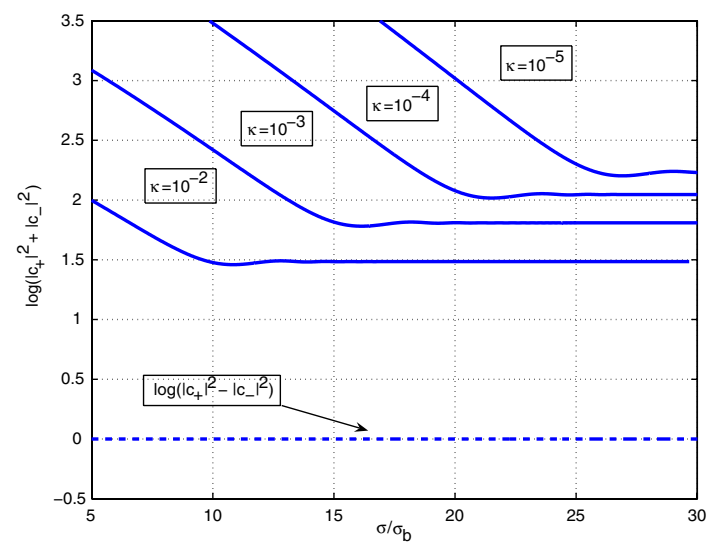

FIG. 3 (color online). In the plot at the left, the numerical result of the spectrum of the canonical momenta (thick lines), $\delta_{\Pi}$ is compared with the analytical expectation (thin lines) for different values of the wave numbers. In the right plot, the numerical evaluation of the mixing coefficients reported for $\kappa \ll 1$ [see Eqs. (3.18) and (3.19)]. In both plots the logarithm (to base 10) of the corresponding function is illustrated as a function of $\sigma$ in units of bouncing time $\sigma_{\mathrm{b}}$. The background used for this integration corresponds to the case $\alpha=4, \phi_{\mathrm{b}}=0$ and $\sigma_{1}=\sigma_{2}=0$. 
reported in terms of the mixing coefficients. We checked that, within the accuracy of the algorithm, the system of Eqs. (3.23), (3.24), and (3.25) with initial conditions given by Eq. (3.26) reproduces the same asymptotic results for the mixing coefficients. From the analytical estimate the spectrum of the mixing coefficients, in the limit $\sigma \gg \sigma_{\mathrm{b}}$, leads to

$$
\left|c_{+}(k)\right|^{2}+\left|c_{-}(k)\right|^{2} \simeq \frac{11}{4}+\frac{25}{16} \ln ^{2} \kappa
$$

that agrees with the explicit numerical calculation of the same quantity reported in Fig. 3. This statement can be explicitly verified by inserting different values of $\kappa$ and by comparing the obtained results with numerical values illustrated in the right plot of Fig. 3. For instance, for $\kappa=$ $10^{-2}$ we get, from Eq. (3.30), $\log \left[\left|c_{+}(k)\right|^{2}+\left|c_{-}(k)\right|^{2}\right] \simeq$ 1.55 , for $\kappa \sim 10^{-5}$ we get $\log \left[\left|c_{+}(k)\right|^{2}+\left|c_{-}(k)\right|^{2}\right] \simeq 2.13$ and so on.

While the right plot of Fig. 3 nicely illustrates the case $\kappa \ll 1$, Fig. 4 deals with the case $\kappa \simeq 1$. In this regime, the mixing coefficient $c_{-}(k)$ is known to be exponentially suppressed. It is clear that from the plot at the right that as $\kappa$ approaches 1 the mixing is suppressed in such a way that for $\kappa \sim 1$ we have that $\left(\left|c_{+}(k)\right|^{2}+\left|c_{-}(k)\right|^{2}\right) \simeq$ $\left(\left|c_{+}(k)\right|^{2}-\left|c_{-}(k)\right|^{2}\right)$ implying that $\left|c_{-}(k)\right|^{2} \simeq 0$.

A relevant implication of the results reported so far is that since the spectrum of the mixing coefficients is logarithmically increasing with the wave number (for $\kappa=$ $\left.k \sigma_{\mathrm{b}}<1\right)$, the spectra of the field operators i.e. $\delta_{h} \simeq$ $k^{3 / 2}\left|F_{k}(\sigma)\right|$ (related to the Fourier transform of the twopoint function at equal times) will be violet (up to logarithmic corrections). By parametrizing the spectrum as $\delta_{h} \simeq k^{\left(n_{\mathrm{t}}-1\right) / 2}$ we have, in the example discussed so far, $n_{\mathrm{t}}=4$ (up to logarithmic corrections). Moreover, since the spectrum of the mixing coefficients increases with frequency it is plausible to expect that the energy spectrum will be even steeper as a function of the wave number $k$. Consequently, the energy spectrum will be convergent in the infrared. In the ultraviolet, the maximally amplified $\kappa$,

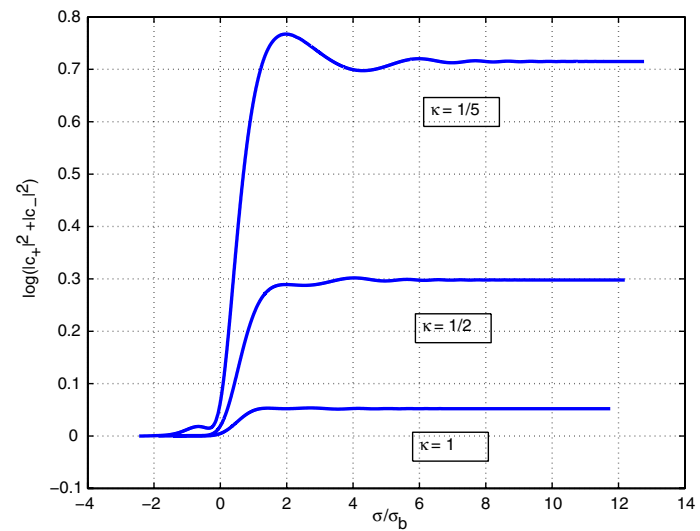

i.e. $\kappa \sim 1$ will give a natural ultraviolet cutoff of the energy density when the (initial) zero-point energy is appropriately subtracted.

It is finally appropriate to notice that the calculation of the mixing coefficients can be conducted also in the string frame. In this case the relevant action will be the one defined in Eq. (1.9). The numerical values of the mixing coefficients as well as their dependence upon the comoving wave number turn out to be exactly the same.

\section{Energy and pressure densities of relic gravitons}

Different possibilities for assigning the energy and pressure densities of the relic gravitons will now be examined. The first strategy is to compute the second-order corrections to the Einstein tensor $\mathcal{G}_{\mu}^{\nu}$ that appears in Eq. (A3). The details of the calculation are reported in the appendix and the main result for the components of the energymomentum pseudotensor can be expressed, in the conformal time parametrization, as follows:

$$
\begin{gathered}
\mathcal{T}_{0}^{0}=\frac{1}{a^{2} \ell_{\mathrm{P}}^{2}}\left[\mathcal{H} h_{k \ell}^{\prime} h^{k \ell}+\frac{1}{8}\left(\partial_{m} h_{k \ell} \partial^{m} h^{k \ell}+h_{k \ell}^{\prime} h^{k \ell \prime}\right)\right], \\
\mathcal{T}^{j}{ }_{i}=\frac{\mathcal{T}}{3} \delta_{i}^{j}+\Sigma_{i}^{j},
\end{gathered}
$$

where

$$
\begin{aligned}
\mathcal{T} & =\frac{1}{a^{2} \ell_{\mathrm{P}}^{2}}\left[\frac{5}{8} h_{k \ell}^{\prime} h^{k \ell \prime}-\frac{7}{8} \partial_{m} h_{k \ell} \partial^{m} h^{k \ell}\right], \\
\Sigma_{i}^{j}= & \frac{1}{a^{2} \ell_{\mathrm{P}}^{2}}\left\{\frac{1}{6}\left[h_{k \ell}^{\prime} h^{k \ell \prime}-\frac{1}{2} \partial_{m} h_{k \ell} \partial^{m} h^{k \ell}\right] \delta_{i}^{j}\right. \\
& \left.+\frac{1}{2} \partial_{m} h_{\ell j} \partial^{m} h^{\ell j}-\frac{1}{4} \partial_{i} h_{k \ell} \partial^{j} h^{k \ell}-\frac{1}{2} h_{k i}^{\prime} h^{k j \prime}\right\},
\end{aligned}
$$

with $\sum_{i}^{i}=0$. From Eqs. (3.31) and (3.33) the components

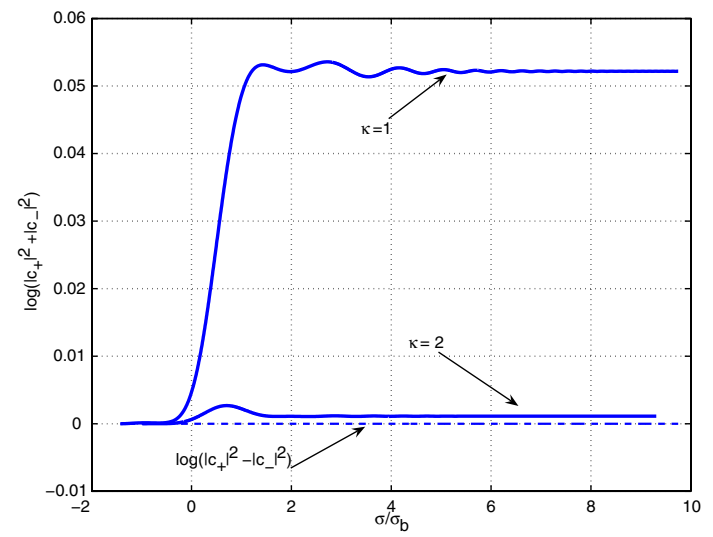

FIG. 4 (color online). The suppression of the mixing coefficients is illustrated for the regime $\kappa>1$. It is clear from both plots that when $\kappa$ increases from $\kappa \leq 1$ to $\kappa>1,\left|c_{-}(k)\right|^{2} \rightarrow 0$. The background parameters are the same as in Fig. 3 . 
of the energy and pressure density can be easily obtained since, by definition, $\rho_{\mathrm{gw}}=\mathcal{T}_{0}^{0}$ and $p_{\mathrm{gw}}=-\mathcal{T} / 3$.

The components of the energy-momentum pseudotensor given in Eqs. (3.31) and (3.32) are not covariantly conserved. However, since the Bianchi identity $\nabla_{\mu} G_{\nu}^{\mu}=0$ should be valid to all orders, we will also have that:

$$
\delta_{\mathrm{t}}^{(2)}\left(\nabla_{\mu} G_{\nu}^{\mu}\right)=0,
$$

whose explicit form is

$\partial_{\mu} \delta_{\mathrm{t}}^{(2)} \mathcal{G}_{\nu}^{\mu}+\delta_{\mathrm{t}}^{(2)} \Gamma_{\mu \alpha}^{\mu} \overline{\mathcal{G}}_{\nu}^{\alpha}+\bar{\Gamma}_{\mu \alpha}^{\mu} \delta_{\mathrm{t}}^{(2)} \mathcal{G}_{\nu}^{\alpha}+\delta_{\mathrm{t}}^{(1)} \Gamma_{\mu \alpha}^{\mu} \delta_{\mathrm{t}}^{(1)} \mathcal{G}_{\nu}^{\alpha}$

$-\delta_{\mathrm{t}}^{(2)} \Gamma_{\nu \alpha}^{\beta} \overline{G_{\beta}^{\alpha}}-\bar{\Gamma}_{\nu \alpha}^{\beta} \delta_{\mathrm{t}}^{(2)} G_{\beta}^{\alpha}-\delta_{\mathrm{t}}^{(1)} \Gamma_{\nu \alpha}^{\beta} \delta_{\mathrm{t}}^{(1)} G_{\beta}^{\alpha}=0$.

Recalling now the components of the energy-momentum pseudotensor and the results for the fluctuations of the Christoffel symbols (i.e. Eqs. (B3) of the appendix) we have

$$
\frac{\partial \rho_{\mathrm{gw}}}{\partial \tau}+3 \mathcal{H}\left(\rho_{\mathrm{gw}}+p_{\mathrm{gw}}\right)-\frac{2\left(\mathcal{H}^{2}-\mathcal{H}^{\prime}\right)}{a^{2} \ell_{\mathrm{P}}^{2}} \delta_{\mathrm{t}}^{(2)} \Gamma_{k 0}^{k}=0,
$$

that can also be written as

$$
\frac{\partial \rho_{\mathrm{gw}}}{\partial \tau}+3 \mathcal{H}\left(\rho_{\mathrm{gw}}+\mathcal{P}_{\mathrm{gw}}\right)=0
$$

where

$$
\mathcal{P}_{\mathrm{gw}}=p_{\mathrm{gw}}+\frac{\left(\mathcal{H}^{2}-\mathcal{H}^{\prime}\right)}{3 \mathcal{H} a^{2}} h_{k \ell}^{\prime} h^{k \ell} .
$$

Equations (3.31), (3.33), and (3.39) imply that the operators corresponding to the energy and pressure densities are, in the $\sigma$-parametrization,

$$
\begin{gathered}
\hat{\rho}_{\mathrm{gw}}=\frac{1}{a^{6}}\left\{4 \mathcal{F}[\hat{h} \hat{\Pi}+\hat{\Pi} \hat{h}]+a^{4} \partial_{m} \hat{h} \partial^{m} \hat{h}+\hat{\Pi}^{2}\right\}, \\
\hat{p}_{\mathrm{gw}}=\frac{1}{3 a^{6}}\left[-5 \hat{\Pi}^{2}+7 a^{4}\left(\partial_{m} \hat{h}\right)\left(\partial^{m} \hat{h}\right)\right], \\
\hat{\mathcal{P}}_{\mathrm{gw}}=\hat{p}_{\mathrm{gw}}+\frac{4}{3 a^{6}}\left(\mathcal{F}-\frac{d \ln \mathcal{F}}{d \sigma}\right)[\hat{\Pi} \hat{h}+\hat{h} \hat{\Pi}],
\end{gathered}
$$

where $\hat{h}$ and $\hat{\Pi}$ are the canonical field operators defined in Eq. (3.5). The averaged components of the energymomentum pseudotensor can be obtained by taking the expectation values of the operators defined in Eqs. (3.40), (3.41), and (3.42) and by recalling that the initial state is the one annihilated by the creation and destruction operators of Eqs. (3.6) and (3.7). The result is then expressed in terms of the appropriate mode functions, i.e.

$$
\begin{aligned}
\left\langle\hat{\rho}_{\mathrm{gw}}\right\rangle= & \frac{1}{a^{6}} \int \frac{d^{3} k}{(2 \pi)^{3}}\left\{4 \mathcal{F}\left[F_{k}(\sigma) G_{k}^{*}(\sigma)+F_{k}^{*}(\sigma) G_{k}(\sigma)\right]\right. \\
& \left.+\Omega^{2}\left|F_{k}(\sigma)\right|^{2}+\left|G_{k}(\sigma)\right|^{2}\right\},
\end{aligned}
$$

$$
\left\langle\hat{p}_{\mathrm{gw}}\right\rangle=\frac{1}{3 a^{6}} \int \frac{d^{3} k}{(2 \pi)^{3}}\left[7 \Omega^{2}\left|F_{k}(\sigma)\right|^{2}-5\left|G_{k}(\sigma)\right|^{2}\right],
$$

$$
\begin{aligned}
\left\langle\hat{\mathcal{P}}_{\mathrm{gw}}\right\rangle= & \left\langle\hat{p}_{\mathrm{gw}}\right\rangle+\frac{4}{3 a^{6}} \int \frac{d^{3} k}{(2 \pi)^{3}}\left(3 \mathcal{F}-\frac{d \ln \mathcal{F}}{d \sigma}\right) \\
& \times\left[F_{k}(\sigma) G_{k}^{*}(\sigma)+F_{k}^{*}(\sigma) G_{k}(\sigma)\right] .
\end{aligned}
$$

It is relevant to remark here that Eq. (3.8) implies that $\partial_{\sigma}\left\langle\hat{\rho}_{\mathrm{gw}}\right\rangle+3 \mathcal{F}\left\langle\hat{\rho}_{\mathrm{gw}}+\hat{\mathcal{P}}_{\mathrm{gw}}\right\rangle=0$. The initial conditions for the field operators (3.6) and (3.7) for $\sigma_{\mathrm{i}} \rightarrow-\infty$ imply that the contribution of the zero-point energy is given by $\int\left(k^{4}\right) /\left(2 \pi^{2} a^{4}\right) d \ln k$ (where we recalled that $\Omega=k a^{2}$ ). To discard this quantity by appropriate subtraction (both in the energy and pressure densities) amounts to neglect the contribution of the zero-point oscillations of the vacuum. As already mentioned, there are different ansatz for the energy-momentum pseudotensor that have been proposed in order to treat backreaction effects of the relic gravitons. It is therefore appropriate to comment here about these possibilities. By looking at the form of Eq. (3.2), the authors of Ref. [38] (see also [39]) argued that a natural ansatz for the energy and pressure densities of the relic gravitons is the one we can derive from the energymomentum tensor of a minimally coupled scalar field for each of the two tensor polarizations. This consideration implies that the energy and pressure densities can be written as

$$
\begin{aligned}
& \hat{\rho}_{\mathrm{gw}}=\frac{1}{a^{6}}\left[\hat{\Pi}^{2}+a^{4} \partial_{m} \hat{h} \partial^{m} \hat{h}\right], \\
& \hat{p}_{\mathrm{gw}}=\frac{1}{a^{6}}\left[\hat{\Pi}^{2}-\frac{a^{4}}{3} \partial_{m} \hat{h} \partial^{m} \hat{h}\right] .
\end{aligned}
$$

The averaged energy and pressure densities become then:

$$
\begin{aligned}
& \left\langle\hat{\rho}_{\mathrm{gw}}\right\rangle=\frac{1}{a^{6}} \int \frac{d^{3} k}{(2 \pi)^{3}}\left[\left|G_{k}(\sigma)\right|^{2}+\Omega^{2}\left|F_{k}(\sigma)\right|^{2}\right], \\
& \left\langle\hat{p}_{\mathrm{gw}}\right\rangle=\frac{1}{a^{6}} \int \frac{d^{3} k}{(2 \pi)^{3}}\left[\left|G_{k}(\sigma)\right|^{2}-\frac{\Omega^{2}}{3}\left|F_{k}(\sigma)\right|^{2}\right] .
\end{aligned}
$$

Equations (3.48) and (3.49) imply $\partial_{\sigma}\left\langle\hat{\rho}_{\mathrm{gw}}\right\rangle+3 \mathcal{F}\left\langle\hat{\rho}_{\mathrm{gw}}+\right.$ $\left.\hat{p}_{\mathrm{gw}}\right\rangle=0$ provided the evolution equations of $F_{k}$ and $G_{k}$ are the ones given in Eq. (3.8).

In order to keep track of the possible ambiguities related with the definitions of the energy-momentum pseudotensor we will first perform the calculations within the approach defined by Eqs. (3.43), (3.44), and (3.45). Then, for each dynamical quantity, the results will be compared with the answers obtained using the approach defined by Eqs. (3.48) and (3.49). 

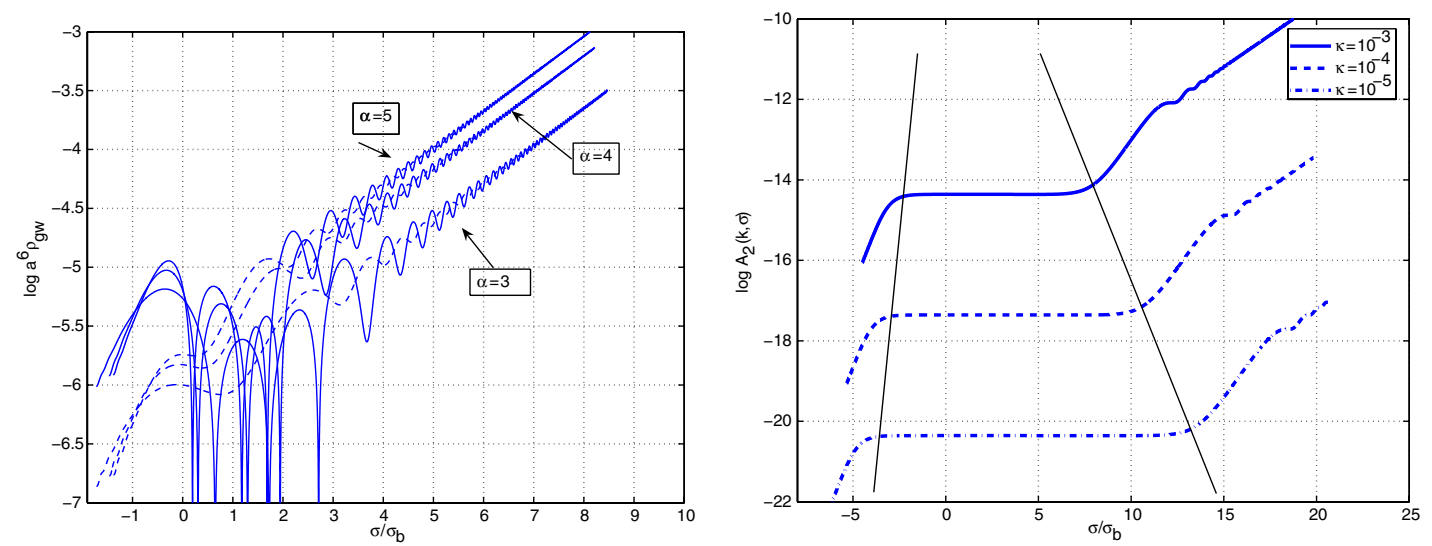

FIG. 5 (color online). Numerical evaluation (left plot) of the integrated energy density for different values of $\alpha$. The contribution of a sample of $k$-modes for the case $\alpha=4$. Initial conditions for the background are fixed in such a way that $\sigma_{1}=\sigma_{2}=0$, while the gauge coupling at the bounce is of order 1 . The initial conditions for the tensor modes are the ones dictated by quantum mechanics (see Sec. III).

\section{EFFECTIVE BAROTROPIC INDICES}

As a consequence of the dynamical evolution of the background the energy and pressure densities have a steep (violet) spectrum. Using the results of the previous section, it appears, for instance that for $k<k_{\max }$ we will have, qualitatively, that the logarithmic spectrum of the energy density is ${ }^{9}$

$$
a^{4} \frac{d \rho_{\mathrm{gw}}}{d \ln k} \simeq k^{4}\left|c_{-}(k)\right|^{2},
$$

where the initial zero-point energy has been already subtracted. For $k>k_{\max }$ the mixing coefficient is not enhanced logarithmically, but rather exponentially suppressed (see Fig. 4 where this aspect is numerically illustrated). Consequently it is plausible that the integrals appearing in the averaged energies and pressure densities are dominated by the modes that are maximally amplified, i.e. $\kappa \simeq \kappa_{\max }$. Since $\kappa_{\max } \simeq 1, k_{\max }=1 / \sigma_{\mathrm{b}}$ (see Fig. 4). The direct calculation supports this view since integrals of the energy density can be performed numerically. Let us then define, for practical reasons,

$$
a^{6} \rho_{\mathrm{gw}}=\int^{k_{\max }} \mathcal{A}_{1,2}(k, \sigma) d \ln k
$$

where the indices 1 and 2 refer to the two different parametrizations of the energy density of the relic gravitons discussed, respectively, in Eqs. (3.43) and (3.48). In Fig. 5 (left plot) the numerical evaluation of $\rho_{\mathrm{gw}}$ is reported. The initial conditions for the mode functions are the ones specified in the previous section for each $k$-mode. With the dashed line the energy density discussed in Eq. (3.48) is illustrated. With the full line the energy density of

\footnotetext{
${ }^{9}$ In the following sections, for sake of simplicity, the averages $\left\langle\hat{\rho}_{\mathrm{gw}}\right\rangle$ will be denoted simply by $\rho_{\mathrm{gw}}$ and similarly for the pressure densities.
}

Eq. (3.43) is instead reported. The integral appearing in Eq. (4.2) is indeed dominated by the upper limit. This aspect is also illustrated, in a related perspective from the right plot of Fig. 5 where the contribution of the integrand for $\kappa \ll 1$ is reported for a sample of $k$-modes. In practice, it is useful to specify a given $\kappa_{\min }$, i.e. the lower limit of integration appearing in Eq. (4.2). For instance, typical values chosen for the present examples are $\kappa=10^{-12}$ or $\kappa=10^{-10}$. Since the spectrum is dominated by $\kappa_{\max }$, different choices $\kappa_{\min }$ produce the same results for the integrated energy density. According to Eq. (3.13), $\kappa_{\min }$ determines a minimal $\sigma_{\text {ex }}$, i.e. $\sigma_{\text {ex }}\left(\kappa_{\min }\right)$. This implies that, to be consistent, the initial conditions for the field operators have been given for $\sigma_{\mathrm{i}} \ll \sigma_{\mathrm{ex}}\left(\kappa_{\min }\right)$ so that, at the initial time, all the tensor modes were of short wavelength.

The right panel of Fig. 5 has some interesting features that will now be discussed. Within the region limited by the two diagonal lines the corresponding modes satisfy $|\kappa \tau|<$ 1. Bearing in mind Eq. (4.2), we then have that for modes of long wavelength $a^{6} \rho_{\mathrm{gw}}$ is constant. Outside the region marked by the two diagonal lines the corresponding modes satisfy $|\kappa \tau|>1$. In this region $a^{6} \rho_{\mathrm{gw}}$ increases as $a^{2}$, i.e. $\rho_{\mathrm{gw}} \sim a^{-4}$.

These considerations suggest that in the short wavelength limit the effective barotropic index is the one of radiation, i.e. $1 / 3$. In fact, in the short wavelength limit the approximate evolution of the mode functions is simply given by Eqs. (3.9) and (3.10). Inserting these solutions either in Eqs. (3.43), (3.44), and (3.45) or in Eqs. (3.48) and (3.49) the results are the same for both parametrizations. In the long wavelength limit, according to Fig. 5 we should instead have that the effective barotropic index is close to 1 .

To corroborate the numerical result, we can solve, directly in the conformal time parametrization, the evolution equations of the mode functions in the approximate background of Eqs. (2.25) and (2.27). The (averaged) energies 
and pressure densities will then be computed and the result expanded in the limit of $k \tau \ll 1$.

Following this procedure [see appendix B and, in particular, Eqs. (C27) and (C28)], from Eqs. (3.48) and (3.49), we have for $\tau<-\tau_{1}$ :

$$
\begin{aligned}
\rho_{\mathrm{gw}} \simeq \frac{1}{a^{4}} \int \frac{k^{4}}{2 \pi^{2}} d \ln k\left[\frac{1}{(-\pi k \tau)}+\mathcal{O}(k \tau)\right], & & \tau<-\tau_{1} \\
p_{\mathrm{gw}} \simeq \frac{1}{a^{4}} \int \frac{k^{4}}{2 \pi^{2}} d \ln k\left[\frac{1}{(-\pi k \tau)}+\mathcal{O}(k \tau)\right], & & \tau<-\tau_{1} .
\end{aligned}
$$

Thus, from Eqs. (4.3) we have that, for $k \tau \ll 1, p_{\mathrm{gw}}=$ $\rho_{\mathrm{gw}}$. Furthermore, recalling that, for $\tau<-\tau_{1}, a(\tau) \sim$ $\sqrt{-\tau}$, we have as expected that $\rho_{\mathrm{gw}}$ and $p_{\mathrm{gw}}$ scale as $a^{-6}$.

If the energy-momentum pseudotensor is derived from the quadratic corrections to the Einstein tensor, the ratio between the pressure and the energy densities becomes, for $k \tau \ll 1$,

$$
\frac{\mathcal{P}_{\mathrm{gw}}}{\rho_{\mathrm{gw}}}=1-\frac{5}{3} \frac{1}{[1-4 \gamma+4 \ln 2-4 \ln (-k \tau)]},
$$

where $\gamma$ is the Euler-Mascheroni constant. Notice that this result is consistent with the one of Eqs. (4.3) with the difference that in Eq. (4.4) logarithmic corrections do appear.

The conclusion previously drawn in the present section, becomes therefore physically justified. The ratio between the energy (or pressure) density of the relic gravitons and the energy (or pressure) density of the dilatonic sources is constant in the long wavelength limit. Of course this conclusion depends on the background. While different backgrounds may change the effective barotropic index of the long wavelengths, the equation of state of the relic gravitons in the limit $k \tau>1$ is always the one of radiation.

It is therefore instructive to compare the present case with the one of a sudden transition from a de Sitter stage of expansion to a radiation-dominated stage of expansion. The typical time scale $\tau_{1}$ will now mark the transition from a de Sitter stage of expansion to a radiationdominated epoch and the analytical form of the scale factor in the two regions is:

$$
\begin{gathered}
a(\tau)=-\frac{\tau_{1}}{\tau}, \quad \tau \leq-\tau_{1}, \\
a(\tau)=\frac{\tau+2 \tau_{1}}{\tau_{1}}, \quad \tau>-\tau_{1} .
\end{gathered}
$$

In this case (see appendix B for further details), Eqs. (3.43), (3.44), and (3.45) imply, for $\tau<-\tau_{1}$,

$$
\begin{gathered}
\rho_{\mathrm{gw}}=\frac{1}{a^{4}} \int \frac{k^{4}}{2 \pi^{2}} d \ln k\left[1-\frac{7}{2 x^{2}}\right], \\
\mathcal{P}_{\mathrm{gw}}=p_{\mathrm{gw}}=\frac{1}{3 a^{4}} \int \frac{k^{4}}{2 \pi^{2}} d \ln k\left[1+\frac{7}{2 x^{2}}\right],
\end{gathered}
$$

where $x=k \tau$. The energy-density is positive for $k \tau \gg 1$ but it becomes negative when the relevant modes become larger than the Hubble radius, i.e. for $k \tau \ll 1$. The equation of state has an effective barotropic index $1 / 3$, for $k \tau \gg 1$. In the opposite limit, i.e. $k \tau \ll 1$ the effective barotropic index becomes $-1 / 3$. From Eqs. (4.7) and (4.8) the energy and pressure densities scale as $a^{-2}$ and this is consistent with Eq. (3.38) for the equation of state $\mathcal{P}_{\mathrm{gw}}=$ $-\rho_{\mathrm{gw}} / 3$.

Consider then the case of modes $k \tau \ll 1$ for $\tau>-\tau_{1}$ i.e. during the radiation-dominated epoch. The exact results are reported in the appendix and here only the long wavelength limit will be given. As the approach defined by Eqs. (3.33) and (3.34) is concerned, the relevant results are reported in Eqs. (C11), (C13), and (C14) of the appendix. To lowest order in $|k \tau| \ll 1$ and $\left|k \tau_{1}\right| \ll 1$ Eq. (C11) leads to

$$
\rho_{\mathrm{gw}}=-\frac{5}{6} \frac{1}{a^{2}} \int \frac{d \ln k}{2 \pi^{2} \tau_{1}^{4}} k^{2} .
$$

In the same limit, from Eqs. (C13) the following result

$$
p_{\mathrm{gw}}=\frac{7}{6} \frac{1}{a^{2}} \int \frac{d \ln k}{2 \pi^{2} \tau_{1}^{4}} k^{2},
$$

can be obtained. We can immediately notice that $p_{\mathrm{gw}} / \rho_{\mathrm{gw}}=-\frac{7}{5}$, which agrees with the calculation of $[35,36]$. The fact that the averaged energy density can have negative values for a limited amount of time was noticed, in a related context, in [58].

Finally, from Eq. (C14), to lowest order in $|x| \ll 1$ and $\left|x_{1}\right| \ll 1$,

$$
\mathcal{P}_{\mathrm{gw}}-p_{\mathrm{gw}}=-\frac{8}{9} \frac{1}{a^{2}} \int \frac{d \ln k}{2 \pi^{2} \tau_{1}^{4}} k^{2} .
$$

Therefore, putting together the results expressed in Eqs. (4.9), (4.10), and (4.11), the following chain of equalities holds

$$
\begin{gathered}
p_{\mathrm{gw}}=-\frac{7}{5} \rho_{\mathrm{gw}}, \\
\mathcal{P}_{\mathrm{gw}}=-\frac{7}{5} \rho_{\mathrm{gw}}+\frac{16}{15} \rho_{\mathrm{gw}}=-\frac{1}{3} \rho_{\mathrm{gw}} .
\end{gathered}
$$

These results coincide with the ones obtained in $[35,36]$.

Instead of using the energy-momentum pseudotensor derived from the quadratic corrections to the Einstein tensor, we could use the energy and pressure densities 
discussed in Eqs. (3.46) and (3.47). The relevant results, in this case, are reported in Eqs. (C16) and (C17) of the appendix. Again, to lowest order in $|x| \ll 1$ and $\left|x_{1}\right| \ll$ 1, Eqs. (C16) and (C17) lead to

$$
\begin{gathered}
\rho_{\mathrm{gw}}=\frac{1}{2 a^{4}} \int \frac{k^{4}}{2 \pi^{2}} d \ln k \frac{x^{2}}{x_{1}^{4}}\left[1-\frac{2}{9} x^{2}+\ldots\right], \\
p_{\mathrm{gw}}=-\frac{1}{6 a^{4}} \int \frac{k^{4}}{2 \pi^{2}} d \ln k \frac{x^{2}}{x_{1}^{4}}\left[1-\frac{6}{9} x^{2}+\ldots\right],
\end{gathered}
$$

where the ellipses stand for higher-order corrections. While in the case of Eq. (4.9) the averaged energy density is negative, in the case of Eq. (4.14) the averaged energy density is positive. Moreover, from Eqs. (4.14) and (4.15) we have that the effective equation of state in the long wavelength limit is given by

$$
p_{\mathrm{gw}}=-\frac{1}{3} \rho_{\mathrm{gw}},
$$

which is the same of Eq. (4.13).

Therefore, we can conclude that both in the bouncing case and in the transition from de Sitter to radiation the two parametrizations of the energy-momentum pseudotensor give the same information. In particular, $\mathcal{P}_{\mathrm{gw}}$ defined by means of the corrections to the Einstein tensor obeys the same equation of state obeyed, in the approach of Eqs. (3.46) and (3.47) by $p_{\mathrm{gw}}$.

\section{ITERATIVE CALCULATIONS OF BACKREACTION EFFECTS}

The evolution equations including the dynamical effects of the produced gravitons reduce to an integro-differential system whose numerical solution may be obtained by means of iterative methods that we are now going to describe and exploit. One of the important qualitative features of the bounce solutions discussed in Sec. II was expressed by the Eq. (2.5) whose form becomes now

$$
\frac{d}{d \sigma}\left(\mathcal{F}+\frac{\xi}{2}\right)=\frac{a^{6}}{4}\left(\rho_{\mathrm{gw}}-p_{\mathrm{gw}}\right),
$$

where with $\rho_{\mathrm{gw}}$ and $p_{\mathrm{gw}}$ we denote the averages of the components of the energy-momentum pseudotensor of the relic gravitons. To this equation the evolution equation for $\xi$ should be added, i.e.

$$
\frac{d \xi}{d \sigma}=-e^{\phi} a^{6}\left[V-\frac{\partial V}{\partial \bar{\phi}}\right]
$$

which is (formally) independent on the energy and pressure densities of the created gravitons. The dynamical quantities appearing in Eq. (5.1) and (5.2) are subjected to the following (generalized) Hamiltonian constraint:

$$
\mathcal{F}^{2}=\frac{a^{6}}{6} \rho_{\mathrm{gw}}+\frac{\xi^{2}}{12}+\frac{e^{\phi} a^{6}}{6} V,
$$

that has to be satisfied along the different steps of the numerical solution. Recalling the examples given in Fig. 5, $\rho_{\mathrm{gw}}$ and $p_{\mathrm{gw}}$ are computed by integrating the evolution equations of the mode functions, i.e. Eqs. (3.8). In this sense, Eq. (5.1) is actually an integro-differential equation whose explicit form is indeed

$$
\frac{d}{d \sigma}\left[\mathcal{F}+\frac{\xi}{2}\right]=\frac{1}{4} \int^{k_{\max }} \mathcal{B}(\Omega, \sigma) d \ln k,
$$

where the quantity $\mathcal{B}(\Omega, \sigma)$ depends on the specific form of the energy-momentum pseudotensor. In particular, subtracting Eq. (3.49) from Eq. (3.48)

$$
\mathcal{B}_{2}(\Omega, \sigma)=\frac{2 k^{3}}{3 \pi^{2}}\left[\Omega^{2}(\sigma)\left|F_{k}(\sigma)\right|^{2}-\Omega(\sigma)\right] .
$$

Similarly, taking the difference between Eq. (3.43) and (3.44)

$$
\begin{aligned}
\mathcal{B}_{1}(\Omega, \sigma)= & \frac{2 k^{3}}{3 \pi^{2}}\left\{2\left|G_{k}(\sigma)\right|^{2}-\Omega^{2}(\sigma)\left|F_{k}(\sigma)\right|^{2}\right. \\
& +3 \mathcal{F}\left[F_{k}(\sigma)^{*} G_{k}(\sigma)+F_{k}(\sigma) G_{k}(\sigma)^{*}\right. \\
& -\Omega(\sigma)]\} .
\end{aligned}
$$

In both equations $F_{k}(\sigma)$ and $G_{k}(\sigma)$ satisfy Eqs. (3.8). Note that in Eqs. (5.5) and (5.6) the zero-point energy and pressure densities have been subtracted as discussed in Sec. IV.

Equation (5.4) can be solved by iteration. ${ }^{10}$ In fact, from Eq. (5.4) the following hierarchy of equations can be deduced:

$$
\begin{gathered}
\mathcal{F}_{0}(\sigma)+\frac{\xi_{0}(\sigma)}{2}=0 \\
\mathcal{F}_{1}(\sigma)+\frac{\xi_{1}(\sigma)}{2}=\frac{1}{4} \int^{\sigma} d \sigma^{\prime} \int_{0}^{k_{\max }} \mathcal{B}_{0}\left(\Omega\left(\sigma^{\prime}\right), \sigma^{\prime}\right) d \ln k
\end{gathered}
$$

$$
\mathcal{F}_{2}(\sigma)+\frac{\xi_{2}(\sigma)}{2}=\frac{1}{4} \int{ }^{\sigma} d \sigma^{\prime} \int_{0}^{k_{k_{\max }}} \mathcal{B}_{1}\left(\Omega\left(\sigma^{\prime}\right), \sigma^{\prime}\right) d \ln k,
$$

and so on. Notice that the subscript appearing in Eqs. (5.8) and (5.9) do not refer to the parametrization of $\mathcal{B}$ [as in Eqs. (5.5) and (5.6)] but to the order of the iteration in spite of the specific choice of $\mathcal{B}$.

The subscripts appearing in the various dynamical quantities of Eqs. (5.7), (5.8), and (5.9) denote the order of the iteration. The source term appearing in the hierarchy is computed from the previous order. So, to zeroth order, i.e. Eq. (5.7), $\mathcal{F}_{0}$ and $\xi_{0}$ are simply the solutions discussed in

\footnotetext{
${ }^{10}$ It should be mentioned that an iterative approach for the solution of the backreaction problems has been also invoked by the authors of Ref. [59].
} 
Sec. II. These solutions will be used to obtain numerically the mode functions that will determine $\mathcal{B}_{0}(\Omega, \sigma)$. To first order, $\mathcal{F}_{1}$ and $\xi_{1}$ will be determined from the zeroth order source, i.e. $\mathcal{B}_{0}(\Omega, \sigma)$. This will lead to the first-order mode functions allowing the quantitative determination of $\mathcal{B}_{1}(\Omega, \sigma)$ and so on.

Based on the results of the previous section, the strongest effect is expected from modes of high wave number (i.e. small wavelength). The rationale for this expectation is that in the long wavelength limit $a^{6}\left(\rho_{\mathrm{gw}}-p_{\mathrm{gw}}\right)$, is smaller than the energy density of the dilaton and also approximately vanishing. In the short wavelength limit $a^{6}\left(\rho_{\mathrm{gw}}-p_{\mathrm{gw}}\right)$ increases as $a^{2}$ and it is expected to dominate the background at sufficiently late times: as time goes by more and more modes will contribute to the ultraviolet branch of the energy spectrum. For the numerical solution of the problem the (stiff) Rosenbrok method [60] has been used. In Fig. 6 the backreaction effects are reported for the case of a zeroth-order solution with $\alpha=4$. With the dashed line the zeroth-order iterative solution is illustrated. With the full line the combined effect of the first-order iteration is illustrated. In a radiation-dominated Universe the evolution equation obeyed by $\mathcal{F}(\sigma)$ is simply given by

$$
\frac{d \mathcal{F}}{d \sigma}=\mathcal{F}^{2}, \quad \mathcal{F}^{2}=\frac{a^{6}}{4} \rho_{\mathrm{r}}
$$

where $\rho_{\mathrm{r}}$ is a generic radiation fluid with $p_{\mathrm{r}}=\rho_{\mathrm{r}} / 3$. As already noticed in connection with Eq. (2.33) the first of the two relations appearing in Eq. (5.10) implies that the Ricci scalar vanishes in a radiation-dominated Universe. The behavior of the solution for $\sigma \gg \sigma_{\mathrm{b}}$, illustrated by the full lines in Fig. 6, corresponds, indeed to the one of a radiation-dominated Universe in the $\sigma$ parametrization. In this parametrization, in fact $\mathcal{F} \simeq\left(\sigma-\sigma_{*}\right)^{-1}$ and $\mathcal{F} / a$ is constant. This nonstandard evolution of the scale factor becomes more familiar by translating the result from the $\sigma$-parametrization to the $\tau$ parametrization. Recalling, in fact, that $a(\sigma)^{2} d \sigma=d \tau$, we do find that $\tau \simeq\left(\sigma-\sigma_{*}\right)^{-1}$. This occurrence implies, as expected, that $a(\tau) \simeq \tau$ which is the well known result for a radiation-dominated Universe in the conformal time parametrization.

In Fig. 6 the backreaction effects have been implemented by using Eq. (5.5) which comes from Eqs. (3.48) and (3.49). In Fig. 7 the numerical calculation has been instead performed by using Eq. (5.6). By comparing Fig. 6 with Fig. 7 the main visible effect is an enhanced oscillating behavior of the first-order iteration after the bounce. However, the evolution of the scale factor and of $\mathcal{F}$ is clearly the same.

The iterative method described in the present section can be also applied in the conformal time parametrization. We will omit here the technical details but the general idea is to start with the asymptotic solution valid for $\tau \ll-\tau_{1}$ [which is, for instance the one provided by Eqs. (2.25) and (2.27)] and then integrate numerically both for the background and for the fluctuations. The main technical difference between the integration in the $\sigma$ and in the $\tau$ parametrization is the following. In the $\sigma$ parametrization the analytical solution of Eqs. (2.18) and (2.19) can be used in the zeroth-order iteration. In the $\tau$ parametrization already the zeroth order iteration requires numerical treatment. Moreover, in the $\tau$ parametrization the evolution equations for the mode functions are formally different and are the ones reported in Eq. (C20) of the appendix B.

In Fig. 8 the integration of the iterative problem is illustrated in the $\tau$ parametrization. With the dashed line the zeroth-order solution is reported. With the full line the result of the first iteration is illustrated. In the left plot of Fig. 8 the parameters are chosen to be the same ones of Figs. 6 and 7. In the right plot the value of the gauge coupling at the bounce is chosen to be much smaller than 1. This implies that the scale factor after the bounce is much larger than in the case when the gauge coupling is of order 1 at the bounce. This can be understood analytically since the asymptotic zeroth-order solution, i.e. Eq. (2.26),
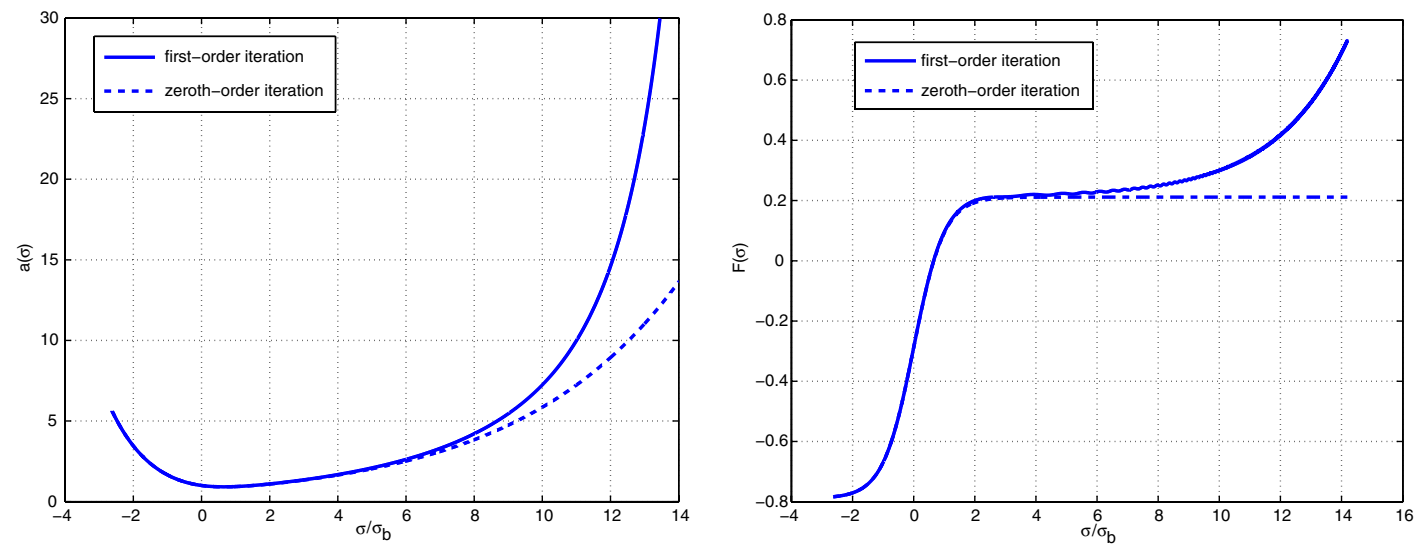

FIG. 6 (color online). The evolution of the scale factor (left plot) and of $\mathcal{F}$ (right plot) in the case $\alpha=4$. The backreaction effects are implemented using the Eq. (5.5). The parameters of the zeroth order solution are fixed by requiring $\sigma_{1}=\sigma_{2}=0$ and $\phi_{\mathrm{b}}=0$. 

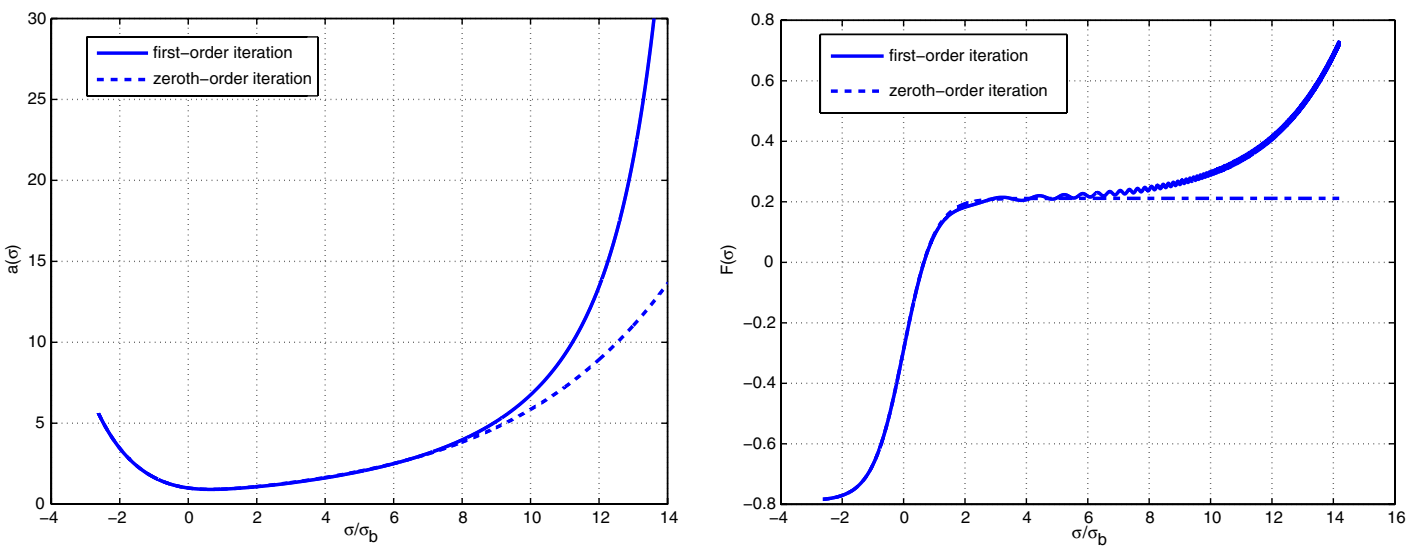

FIG. 7 (color online). The same quantities reported in Fig. 6 but using implementing the backreaction effects through the energy and pressure densities stemming from the quadratic corrections to the Einstein tensor [i.e. Eq. (5.6)].
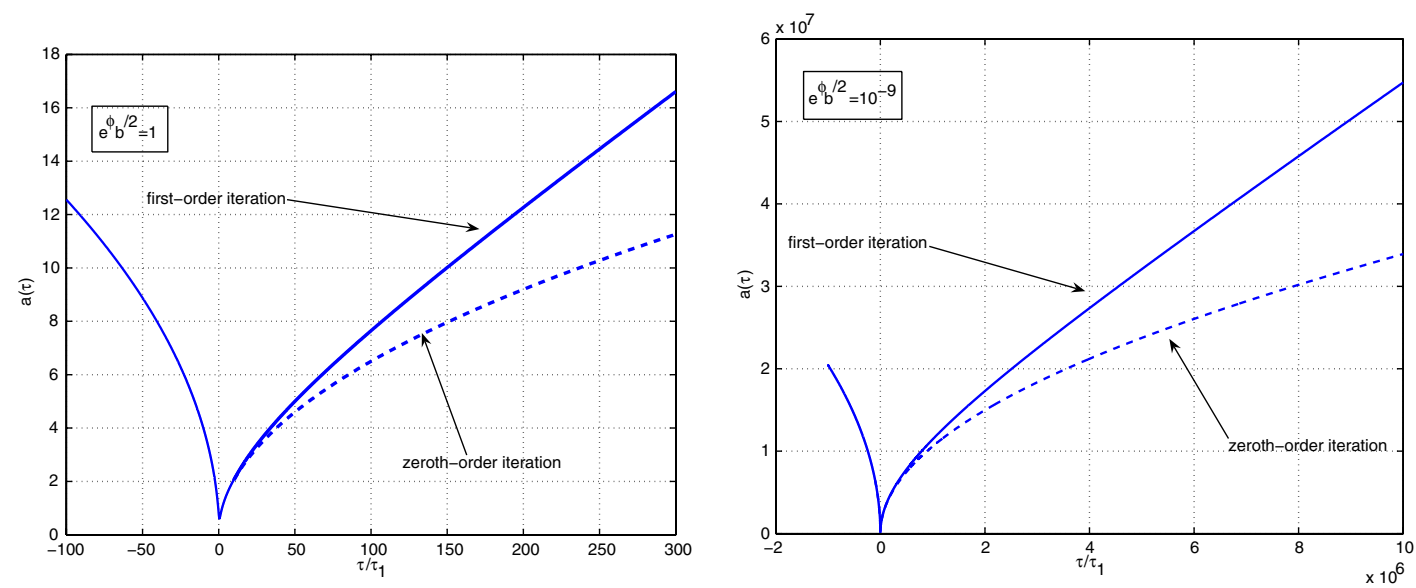

FIG. 8 (color online). Integration of the iterative problem in the conformal time parametrization. In the left plot the parameters of the zeroth-order solution coincide (in the limit $\tau \rightarrow-\infty$ ) with the parameters chosen for the integrations reported in Figs. 6 and 7 (for $\sigma \rightarrow-\infty)$.

leads to a scale factor whose absolute normalization increases as the gauge coupling at the bounce diminishes. It is evident from Fig. 8 that the first-order iteration already leads to a scale factor that increases linearly in conformal time. For both plots in Fig. 8 the backreaction effects have been parametrized through the analog of Eq. (5.5), appropriately translated in the $\tau$ parametrization.

Before closing the present section, it is appropriate to discuss the second and higher order in the iteration scheme presented here. The effect of the second iteration leads to results that are typically smaller than the first iteration by a factor $1 / a^{-2}$. Since the first iteration, as numerically demonstrated, leads to an expanding (radiation-dominated) Universe the effect of the second iteration leads to a comparatively small correction that becomes even smaller as time goes by. Of course, this discussion holds in the case of the class of bouncing models investigated in the present paper. Different physical situations may lead to opposite conclusion on the validity of the iteration scheme discussed in the present paper. We believe, however, that the iteration scheme proposed in the present paper may be usefully employed also in other (related) contexts.

\section{CONCLUDING REMARKS}

Dynamical backreaction of relic gravitons is relevant in different contexts, so that the nature of the induced physical effects may vary. A particularly interesting situation is the one of bouncing solutions of pre-big bang type where backreaction effects are known to be important already from qualitative estimates. One of the purposes of the present investigation is to propose a theoretical scheme where more quantitative predictions could be derived. The present findings are then useful for implementing a smooth 
exit from the pre-big bang phase with consequent production of radiation. The bouncing solutions examined here may also be viewed as a practical theoretical laboratory where the methods for the analysis of the backreaction effects of relic gravitons may be tested. In this respect, the reported results suggest that the role of the produced gravitons is crucial for determining the asymptotic state of the solution at late times.

The strategy followed in the present analysis relies on the interplay between analytical methods and numerical calculations. We have been interested in situations where the Universe undergoes an accelerated contraction before the bounce, while, after the bounce, the zeroth-order solution exhibits a decelerated expansion. After quantizing the tensor modes of the geometry, the initial values of the field operators have been set well before the bounce. At that time all the gravitons were of short-wavelengths in comparison with the typical time scale of the background evolution. Then, the field operators have been evolved in the Heisenberg representation and the mixing coefficients have been accurately determined both analytically and numerically. This step allowed to understand what kind of regularization is required for the energy and pressure densities of the produced gravitons. The second step has been to adopt a consistent ansatz for the gravitational energy-momentum pseudotensor. Two complementary definitions of the energy and pressure densities of the relic gravitons have then been scrutinized and compared both analytically and numerically. On the basis of the reported results, these two different approaches lead to compatible quantitative results.

The self-consistent analysis of backreaction effects leads naturally to an integro-differential problem. Consequently, an iterative method for its solution has been proposed and tested in the specific dynamical framework of bouncing solutions that arise in the context of the low-energy string effective action supplemented by a nonlocal dilaton potential. It turns out that the treatment both of the background and of the tensor fluctuations becomes rather simple by selecting a different parametrization of the time coordinate which does not coincide with the usual conformal or cosmic times. In this time parametrization analytical bouncing solutions can be derived in the Einstein frame metric.

By looking at the structure of the low-energy string effective action, it is possible to infer that not only relic gravitons but also other massless fields may lead to similar effects whose analysis is certainly one of the possible developments of future studies. In this respect, the present investigation dealt with the minimal field content of the model with the aim of achieving a reasonably accurate description of the underlying physical processes. Thus, some of the ideas discussed here will hopefully be useful also for the description of dynamical systems with a richer field content.

\section{APPENDIX A: COVARIANT EQUATIONS WITH NONLOCAL DILATON POTENTIAL}

The problem will be directly analyzed in the Einstein frame metric where the dilaton field and the EinsteinHilbert term are decoupled and the relevant action is [46]

$$
S=\int d^{4} x \sqrt{-g}\left[-\frac{R}{2 \ell_{\mathrm{P}}^{2}}+\frac{1}{2} g^{\alpha \beta} \partial_{\alpha} \phi \partial_{\beta} \phi-W(\bar{\phi})\right],
$$

where

$$
\begin{aligned}
W(\bar{\phi})= & e^{\phi} V\left(e^{-\bar{\phi}}\right), \\
e^{-\bar{\phi}}= & e^{\phi / 2} \int d^{4} y\left(\sqrt{-g} \sqrt{g^{\alpha \beta} \partial_{\alpha} \phi \partial_{\beta} \phi}\right)_{y} \\
& \times \delta(\phi(x)-\phi(y)) .
\end{aligned}
$$

In the following the prime denotes a derivation of the corresponding function with respect to his own argument (so, for instance, $V^{\prime}$ will denote a derivation of $V$ with respect to $e^{-\bar{\phi}}$ and so on).

The evolution equations derived from the action (A1) are then [46]

$$
\begin{gathered}
\mathcal{G}_{\mu}^{\nu}=\ell_{\mathrm{P}}^{2}\left[T_{\mu}^{\nu}(\phi)+\tilde{T}_{\mu}^{\nu}(\phi, g)\right], \\
g^{\alpha \beta} \nabla_{\alpha} \nabla_{\beta} \phi+e^{\phi}\left[V-\frac{1}{2} \frac{\partial V}{\partial \bar{\phi}}\right. \\
\left.-e^{\phi / 2}\left(\frac{\gamma^{\mu \nu} \nabla_{\mu} \nabla_{\nu} \phi}{\sqrt{g^{\alpha \beta} \partial_{\alpha} \phi \partial_{\beta} \phi}} I_{1}-V^{\prime} I_{2}\right)\right]=0,
\end{gathered}
$$

where $G_{\mu}^{\nu}$ is the Einstein tensor already introduced in Eq. (1.2). In Eq. (A3) $T_{\mu}^{\nu}(\phi)$ and $\tilde{T}_{\mu}^{\nu}(\phi, g)$ are, respectively,

$$
\begin{gathered}
T_{\mu}^{\nu}(\phi)=\partial_{\mu} \phi \partial^{\nu} \phi-\frac{1}{2} \delta_{\mu}^{\nu} g^{\alpha \beta} \partial_{\alpha} \phi \partial_{\beta} \phi, \\
\tilde{T}_{\mu}^{\nu}(\phi, g)=e^{\phi}\left(V \delta_{\mu}^{\nu}+\gamma_{\mu}^{\nu} \sqrt{g^{\alpha \beta} \partial_{\alpha} \phi \partial_{\beta} \phi} e^{\phi / 2} I_{1}\right) ;
\end{gathered}
$$

where the induced metric $\gamma_{\mu \nu}$

$$
\gamma_{\mu \nu}=g_{\mu \nu}-\frac{\partial_{\mu} \phi \partial_{\nu} \phi}{g^{\alpha \beta} \partial_{\alpha} \phi \partial_{\beta} \phi},
$$

has been defined. In Eqs. (A4) and (A6) the two integrals $I_{1}$ and $I_{2}$ are:

$$
\begin{aligned}
& I_{1}(x)=\frac{1}{\ell_{\mathrm{P}}^{3}} \int d^{4} y\left(\sqrt{-g} V^{\prime}\right)_{y} \delta(\phi(x)-\phi(y)), \\
& I_{2}(x)=\frac{1}{\ell_{\mathrm{P}}^{3}} \int d^{4} y\left(\sqrt{-g} \sqrt{g^{\alpha \beta} \partial_{\alpha} \phi \partial_{\beta} \phi}\right)_{y} \delta^{\prime}(\phi(x)-\phi(y)) .
\end{aligned}
$$




\section{APPENDIX B: ENERGY-MOMENTUM PSEUDO-TENSOR}

From Eq. (1.1) we have

$$
\delta_{\mathrm{t}}^{(1)} g_{i j}=-a^{2} h_{i j}, \quad h_{i}^{i}=\partial_{i} h_{j}^{i}=0,
$$

where, as in the bulk of the paper, the subscript refers to the tensor nature of the fluctuation while the superscript denotes the perturbative order. Equation (B1) implies that

$$
\delta_{\mathrm{t}}^{(1)} g^{i j}=\frac{h^{i j}}{a^{2}}, \quad \delta_{\mathrm{t}}^{(2)} g^{i j}=-\frac{h_{k}^{i} h^{k j}}{a^{2}} .
$$

Consequently, the fluctuations of the Christoffel connections to first and second order become:

$$
\begin{aligned}
& \delta_{\mathrm{t}}^{(1)} \Gamma_{i j}^{0}=\frac{1}{2}\left(h_{i j}^{\prime}+2 \mathcal{H} h_{i j}\right), \\
& \delta_{\mathrm{t}}^{(1)} \Gamma_{i 0}^{j}=\frac{1}{2} h_{i}^{j \prime}, \\
& \delta_{\mathrm{t}}^{(1)} \Gamma_{i j}^{k}=\frac{1}{2}\left(\partial_{i} h_{j}^{k}+\partial_{j} h_{i}^{k}-\partial^{k} h_{i j}\right), \\
& \delta_{\mathrm{t}}^{(2)} \Gamma_{i 0}^{j}=-\frac{1}{2} h^{i k} h_{k j}^{\prime}, \\
& \delta_{\mathrm{t}}^{(2)} \Gamma_{i j}^{k}=\frac{1}{2} h^{i \ell}\left[\partial_{\ell} h_{j k}-\partial_{k} h_{j \ell}-\partial_{j} h_{k \ell}\right],
\end{aligned}
$$

where, as in the bulk of the paper, the prime denotes a derivation with respect to the conformal time coordinate. The fluctuations of the various components of the Ricci tensor to first and second order are then:

$$
\begin{gathered}
\delta_{\mathrm{t}}^{(1)} R_{i j}=\frac{1}{2}\left[h_{i j}^{\prime \prime}+2 \mathcal{H} h_{i j}^{\prime}-\nabla^{2} h_{i j}\right]+\left(\mathcal{H}^{\prime}+2 \mathcal{H}^{2}\right) h_{i j}, \\
\delta_{\mathrm{t}}^{(2)} R_{00}=\frac{1}{4} h_{i j}^{\prime} h^{i j \prime}-\frac{\mathcal{H}}{2} h_{i j} h^{i j \prime}+\frac{1}{2} h^{i j} \nabla^{2} h_{i j}, \\
\delta_{\mathrm{t}}^{(2)} R_{i j}=\frac{1}{2} h^{k \ell}\left[\partial_{k} \partial_{\ell} h_{i j}-\partial_{k} \partial_{j} h_{\ell i}-\partial_{k} \partial_{i} h_{j \ell}\right]-\frac{1}{2} \partial_{j}\left[h^{k \ell}\left(\partial_{\ell} h_{i k}-\partial_{k} h_{\ell i}-\partial_{i} h_{k \ell}\right)\right]-\frac{\mathcal{H}}{2} h^{k \ell} h_{k \ell}^{\prime} \delta_{i j}+\frac{\mathcal{H}}{2} h_{j}^{\ell} h_{\ell i}^{\prime} \\
+\frac{\mathcal{H}}{2} h_{i}^{\ell} h_{\ell j}^{\prime}-\frac{1}{4} h_{j}^{k \prime} h_{i k}^{\prime}-\frac{\mathcal{H}}{2} h_{j}^{k \prime} h_{i k}-\frac{1}{4} h_{i}^{k \prime} h_{k j}^{\prime}-\frac{\mathcal{H}}{2} h_{i}^{k \prime} h_{k j}-\frac{1}{4}\left[\partial_{i} h_{k}^{\ell}+\partial_{k} h_{i}^{\ell}-\partial^{\ell} h_{i k}\right]\left[\partial_{\ell} h_{j}^{k}+\partial_{j} h_{\ell}^{k}-\partial^{k} h_{j \ell}\right] .
\end{gathered}
$$

The Ricci scalar is zero to first order in the tensor fluctuations, i.e. $\delta_{\mathrm{t}}^{(1)} R=0$. This is due to the traceless nature of these fluctuations. To second-order, however, $\delta_{\mathrm{t}}^{(2)} R \neq 0$ and its form is:

$$
\begin{aligned}
\delta_{\mathrm{t}}^{(2)} R= & \frac{1}{a^{2}}\left\{\frac{3}{4} h_{k \ell}^{\prime} h^{k \ell \prime}+\mathcal{H} h_{k \ell}^{\prime} h^{k \ell}+\frac{1}{2} h^{k \ell} \nabla^{2} h_{k \ell}-\frac{1}{4} \partial_{i} h^{k \ell} \partial^{i} h_{k \ell}\right\}+\frac{1}{a^{2}}\left\{-\frac{1}{2} \partial_{i}\left[h^{k \ell}\left(\partial_{\ell} h_{k}^{i}-\partial_{k} h_{\ell}^{i}-\partial^{i} h_{k \ell}\right)\right]\right. \\
& \left.-\frac{1}{4}\left[\partial_{i} h_{k}^{\ell} \partial_{\ell} h_{i}^{k}-\partial_{i} h_{k}^{\ell} \partial^{k} h_{i \ell}+\partial_{k} h^{\ell i} \partial_{\ell} h_{i}^{k}-\partial^{\ell} h_{i k} \partial^{i} h_{\ell}^{k}+\partial^{\ell} h_{i k} \partial^{k} h_{i \ell}\right]\right\} .
\end{aligned}
$$

Recalling the form of the Einstein tensor [see Eq. (1.2)],

$$
\delta_{\mathrm{t}}^{(2)} G_{00}=-\ell_{\mathrm{P}}^{2} \mathcal{T}_{00}=\delta_{\mathrm{t}}^{(2)} R_{00}-\frac{1}{2} \bar{g}_{00} \delta_{\mathrm{t}}^{(2)} R,
$$

we obtain

$$
\ell_{\mathrm{P}}^{2} \mathcal{T}_{00}=\mathcal{H} h_{k \ell}^{\prime} h^{k \ell}+\frac{1}{8}\left(h_{k \ell}^{\prime} h^{k \ell \prime}+\partial_{i} h_{k \ell} \partial^{i} h^{k \ell}\right)+\mathcal{D}_{00}
$$

where $\mathcal{D}_{00}$ is a total derivative, i.e.

$$
\mathcal{D}_{00}=\frac{1}{8} \partial_{\ell}\left[\partial_{i} h^{k \ell} h_{k}^{i}-2 \partial_{k} h_{i}^{\ell} h^{k i}\right]
$$

From Eqs. (B6) and (B7) it is also possible to write:

$$
\begin{aligned}
\delta_{\mathrm{t}}^{(2)} R_{i j}= & \frac{1}{4}\left(\partial_{k} h_{i}^{\ell} \partial^{k} h_{j \ell}+\partial^{\ell} h_{i k} \partial_{\ell} h_{j}^{k}\right)-\frac{1}{4} \partial_{i} h_{k \ell} \partial_{j} h^{k \ell} \\
& -\frac{\mathcal{H}}{2} h^{k \ell} h_{k \ell}^{\prime} \delta_{i j}+\frac{\mathcal{H}}{2}\left(h_{j}^{\ell} h_{\ell i}^{\prime}+h_{i}^{\ell} h_{\ell j}^{\prime}\right) \\
& -\frac{\mathcal{H}}{2}\left(h_{j}^{k \prime} h_{i k}+h_{i}^{k \prime} h_{k j}\right)-\frac{1}{4}\left(h_{j}^{k \prime} h_{i k}^{\prime}+h_{i}^{k \prime} h_{k j}^{\prime}\right) \\
& +\mathcal{D}_{i j}, \\
\delta_{\mathrm{t}}^{(2)} R= & \frac{1}{a^{2}}\left[\frac{3}{4} h_{k \ell}^{\prime} h^{k \ell \prime}+\mathcal{H} h_{k \ell}^{\prime} h^{k \ell}-\frac{3}{4} \partial_{i} h^{k \ell} \partial^{i} h_{k \ell}\right] \\
& +\frac{1}{a^{2}} \mathcal{D}_{R},
\end{aligned}
$$

where $\mathcal{D}_{i j}$ and $\mathcal{D}_{R}$ are further total derivative 


$$
\begin{aligned}
\mathcal{D}_{i j}= & \frac{1}{2} \partial_{k}\left[h^{k \ell}\left(\partial_{\ell} h_{i j}-\partial_{j} h_{i \ell}-\partial_{i} h_{j \ell}\right)\right] \\
& -\frac{1}{2} \partial_{j}\left[h^{k \ell}\left(\partial_{\ell} h_{i k}-\partial_{k} h_{\ell i}-\partial_{i} h_{k \ell}\right)\right] \\
& -\frac{1}{4} \partial_{\ell}\left[\partial_{k} h_{i}^{\ell} h_{j}^{k}+h_{i k} \partial^{k} h_{j}^{\ell}\right] . \\
\mathcal{D}_{R}= & \partial_{i}\left[h^{k \ell} \partial^{i} h_{k \ell}\right]+\frac{1}{4} \partial_{\ell}\left[\partial_{i} h^{k \ell} h_{k}^{i}-2 \partial_{k} h_{i}^{\ell} h^{k i}\right],
\end{aligned}
$$

Therefore, up to total derivatives, the following result holds:

$$
\ell_{\mathrm{P}}^{2} \mathcal{T}_{00}=\mathcal{H} h_{k \ell}^{\prime} h^{k \ell}+\frac{1}{8}\left(h_{k \ell}^{\prime} h^{k \ell l}+\partial_{i} h_{k \ell} \partial^{i} h^{k \ell}\right),
$$

and

$$
\begin{aligned}
\ell_{\mathrm{P}}^{2} \mathcal{T}_{i j}= & \frac{3}{8} \delta_{i j}\left[\partial_{m} h_{k \ell} \partial^{m} h^{k \ell}-h_{k \ell}^{\prime} h^{k \ell \prime}\right]+\frac{1}{2} h_{j}^{k \prime} h_{i k}^{\prime} \\
& +\frac{1}{4} \partial_{i} h_{k \ell} \partial_{j} h^{k \ell}-\frac{1}{2} \partial_{k} h_{i}^{\ell} \partial^{k} h_{\ell j} .
\end{aligned}
$$

To pass from doubly covariant indices to mixed ones, it is useful to recall that, to second order,

$$
\begin{aligned}
\delta^{(2)} \mathcal{G}_{\mu}^{\nu} & =\delta^{(2)}\left[g^{\nu \alpha} G_{\mu \alpha}\right] \\
& =\delta^{(2)} g^{\nu \alpha} \bar{G}_{\mu \alpha}+\bar{g}^{\nu \alpha} \delta^{(2)} \mathcal{G}_{\mu \alpha}+\delta^{(1)} g^{\nu \alpha} \delta^{(1)} G_{\mu \alpha} .
\end{aligned}
$$

By looking at the form of the specific terms arising in the previous equation it is clear that $\mathcal{T}_{0}^{0}=\bar{g}^{00} \mathcal{T}_{00}$ and that $\mathcal{T}_{i}^{j}=\bar{g}^{j k} \mathcal{T}_{k i}$. The expressions for $\mathcal{T}_{0}^{0}$ and $\mathcal{T}_{i}^{j}$ are the ones reported in Eqs. (3.31) and (3.32) [see also Eqs. (3.33) and (3.34)]. These expressions coincide with the ones obtained, for instance, in $[35,36]$ and are also consistent with the ones of $[33,34]$.

\section{APPENDIX C: ANALYTICAL ESTIMATES OF THE EFFECTIVE BAROTROPIC INDICES}

Since the Hamiltonian defined in Eq. (3.4) is timedependent, it is always possible to perform time-dependent canonical transformations, leading to a different form of the Hamiltonian that will be classically equivalent to (3.4). This procedure correspond to drop total time derivatives from the corresponding classical action [61].

From the action (3.3), defining the new field $\mu=a h$ and dropping a total time derivative the following Hamiltonian can be obtained in the $\tau$-parametrization

$$
\tilde{H}_{\mathrm{gw}}(\tau)=\frac{1}{2} \int d^{3} x\left[\pi^{2}+\left(\partial_{i} \mu\right)^{2}-\left(\mathcal{H}^{2}+\mathcal{H}^{\prime}\right) \mu^{2}\right],
$$

where $\pi=\mu^{\prime}$. This form of the Hamiltonian is particularly convenient for the studying the time evolution of the field operators in the conformal time parametrization. By appropriate Fourier transforms of the field operators [see Eq. (3.5)], the analog of Eqs. (3.6) and (3.7) are

$$
\begin{aligned}
\hat{\mu}_{\vec{k}}(\tau) & =\hat{b}_{\vec{k}}\left(\tau_{\mathrm{i}}\right) f_{k}(\tau)+\hat{b}_{-\vec{k}}^{\dagger}\left(\tau_{\mathrm{i}}\right) f_{k}^{*}(\tau), \\
\hat{\pi}_{\vec{k}}(\tau) & =\hat{b}_{\vec{k}}\left(\tau_{\mathrm{i}}\right) g_{k}(\tau)+\hat{b}_{-\vec{k}}^{\dagger}\left(\tau_{\mathrm{i}}\right) g_{k}^{*}(\tau),
\end{aligned}
$$

where the new mode functions now obey

$$
f_{k}^{\prime \prime}+\left[k^{2}-\frac{a^{\prime \prime}}{a}\right] f_{k}=0, \quad g_{k}=f_{k}^{\prime} .
$$

In the limit $\tau_{\mathrm{i}} \rightarrow-\infty$, the operators $\hat{b}_{\vec{k}}\left(\tau_{\mathrm{i}}\right)$ and $\hat{b}_{-\vec{k}}\left(\tau_{\mathrm{i}}\right)$ annihilate the same initial vacuum state as the one annihilated by $\hat{a}_{\vec{k}}\left(\sigma_{\mathrm{i}}\right)$ and $\hat{a}_{-\vec{k}}\left(\sigma_{\mathrm{i}}\right)$ in the limit $\sigma_{\mathrm{i}} \rightarrow-\infty$.

In this framework all the calculations discussed in the $\sigma$-parametrization can be reformulated. The expectation values of the energy and pressure density can be computed. For instance from Eqs. (3.31) and (3.32) we have ${ }^{11}$

$$
\begin{aligned}
\left\langle\hat{\rho}_{\mathrm{gw}}\right\rangle= & \frac{1}{a^{4}} \int \frac{d^{3} k}{(2 \pi)^{3}}\left\{\left(k^{2}-7 \mathcal{H}^{2}\right)\left|f_{k}(\tau)\right|^{2}+\left|g_{k}(\tau)\right|^{2}\right. \\
& \left.+3 \mathcal{H}\left[f_{k}(\tau) g_{k}^{*}(\tau)+f_{k}^{*}(\tau) g_{k}(\tau)\right]\right\}, \\
\left\langle\hat{p}_{\mathrm{gw}}\right\rangle= & \frac{1}{3 a^{4}} \int \frac{d^{3} k}{(2 \pi)^{3}}\left\{\left|f_{k}(\tau)\right|^{2}\left(7 k^{2}-5 \mathcal{H}^{2}\right)-5\left|g_{k}(\tau)\right|^{2}\right. \\
& \left.+5 \mathcal{H}\left[f_{k}^{*}(\tau) g_{k}(\tau)+f_{k}(\tau) g_{k}^{*}(\tau)\right]\right\} . \\
\left\langle\hat{\mathcal{P}}_{\mathrm{gw}}\right\rangle= & \left\langle\hat{p}_{\mathrm{gw}}\right\rangle+\frac{4\left(\mathcal{H}^{2}-\mathcal{H}^{\prime}\right)}{3 \mathcal{H} a^{4}} \int \frac{d^{3} k}{(2 \pi)^{3}}\left[f_{k}(\tau) g_{k}^{*}(\tau)\right. \\
& \left.+f_{k}^{*}(\tau) g_{k}(\tau)\right] .
\end{aligned}
$$

In similar terms, the expectation values of the energy and pressure densities can be obtained within the approach of Refs. [38,39].

Consider now the case of a transition from a de Sitter stage of expansion to a radiation-dominated stage of expansion, i.e. Eqs. (4.5) and (4.6). For $\tau \leq-\tau_{1}$,

$$
\begin{aligned}
f_{k}(\tau) & =\frac{1}{\sqrt{2 k}}\left(1-\frac{i}{x}\right) e^{-i x}, \\
g_{k}(\tau) & =i \sqrt{\frac{k}{2}}\left[\frac{1}{x^{2}}-1+\frac{i}{x}\right] e^{-i x},
\end{aligned}
$$

where $x=k \tau$. For $\tau>-\tau_{1}$ the canonical fields can be expressed as

$$
\begin{aligned}
\hat{\mu}_{\vec{k}}(\tau) & =\hat{b}_{\vec{k}} F_{k}(\tau)+\hat{b}_{-\vec{k}}^{\dagger} F_{k}^{*}(\tau), \\
\hat{\pi}_{\vec{k}}(\tau) & =\hat{b}_{\vec{k}} G_{k}(\tau)+\hat{b}_{-\vec{k}}^{\dagger} G_{k}^{*}(\tau),
\end{aligned}
$$

where the mode functions are now

$$
F_{k}(\tau)=\frac{1}{\sqrt{2 k}} e^{-i\left(x+2 x_{1}\right)}, \quad G_{k}(\tau)=-i \sqrt{\frac{k}{2}} e^{-i\left(x+2 x_{1}\right)} .
$$

Since the field operators must be continuous we have

\footnotetext{
${ }^{11}$ In the bulk of the paper, for practical reasons, we dropped the expectation values for the averaged quantities. In this appendix, to make clear the computational procedures, the expectation values will be restored.
} 


$$
\begin{aligned}
& f_{k}\left(-\tau_{1}\right)=c_{+}(k) F_{k}\left(-\tau_{1}\right)+c_{-}(k) F_{k}^{*}\left(-\tau_{1}\right), \\
& \tilde{g}_{k}\left(-\tau_{1}\right)=c_{+}(k) G_{k}\left(-\tau_{1}\right)+c_{-}(k) G_{k}^{*}\left(-\tau_{1}\right) .
\end{aligned}
$$

so that $c_{+}(k)$ and $c_{-}(k)$ can be determined as

$$
c_{+}(k)=\frac{e^{2 i x_{1}}\left(2 x_{1}^{2}-1+2 i x_{1}\right)}{2 x_{1}^{2}}, \quad c_{-}(k)=\frac{1}{2 x_{1}^{2}},
$$

where $x_{1}=k \tau_{1}$. It can be verified immediately that $\left|c_{+}(k)\right|^{2}-\left|c_{-}(k)\right|^{2}=1$.

It is now instructive to consider the behavior of the effective barotropic indices in the two regimes $\tau<-\tau_{1}$ and $\tau>-\tau_{1}$. Furthermore, in each regime, we shall be interested in the relative weight of the short and long wavelength modes. This analysis is useful for the comparison with the case of bouncing models. Since during the de Sitter phase, $\mathcal{H}^{\prime}=\mathcal{H}^{2}$, Eqs. (C4) imply that $\left\langle\hat{p}_{\mathrm{gw}}\right\rangle=$ $\left\langle\hat{\mathcal{P}}_{\mathrm{gw}}\right\rangle$. Furthermore, if we will have that $3\left\langle\rho_{\mathrm{gw}}\right\rangle=\left\langle p_{\mathrm{gw}}\right\rangle$. During the radiation-dominated phase, i.e. $\tau>-\tau_{1}$, the short wavelength modes also obey a radiative equation of state and the energy and pressure densities explicitly depend upon the mixing coefficients, i.e.

$$
\begin{aligned}
& \left\langle\hat{\rho}_{\mathrm{gw}}\right\rangle=\frac{1}{a^{4}} \int \frac{k d^{3} k}{(2 \pi)^{3}}\left[2\left|c_{-}(k)\right|^{2}+1\right], \\
& \left\langle\hat{\mathcal{P}}_{\mathrm{gw}}\right\rangle=\left\langle p_{\mathrm{gw}}\right\rangle=\frac{1}{3 a^{4}} \int \frac{k d^{3} k}{(2 \pi)^{3}}\left[2\left|c_{-}(k)\right|^{2}+1\right],
\end{aligned}
$$

where, for simplicity, the vacuum contribution has not been subtracted. Notice that, now (i.e. in the radiationdominated epoch and for $k \tau \gg 1$ ), the equality of $\left\langle\hat{\mathcal{P}}_{\mathrm{gw}}\right\rangle$ and $\left\langle\hat{p}_{\mathrm{gw}}\right\rangle$, is a consequence of the explicit form of the mode functions in this regime, i.e. $F_{k} G_{k}^{*}+F_{k}^{*} G_{k}=0$.

In the long wavelength limit, i.e. $k \tau \ll 1$ and during the de Sitter phase we obtain, instead, the same expressions reported in Eqs. (4.7) and (4.8). Consider finally the last case, i.e. long wavelength modes during the radiationdominated epoch. After making use of the explicit expressions of the mixing coefficients we will have, for the averaged energy density,

$$
\begin{aligned}
\left\langle\hat{\rho}_{\mathrm{gw}}\right\rangle= & \frac{1}{a^{4}} \int \frac{k d^{3} k}{(2 \pi)^{3}}\left\{\frac{1}{2}\left[2-\frac{7}{\left(x+2 x_{1}\right)^{2}}\right]\left(1+\frac{1}{2 x_{1}^{4}}\right)\right. \\
& -\sin 2\left(x+x_{1}\right)\left[\frac{3\left(2 x_{1}^{2}-1\right)}{2\left(x+2 x_{1}\right) x_{1}^{4}}+\frac{7}{2\left(x+2 x_{1}\right)^{2} x_{1}^{3}}\right] \\
& \left.+\cos 2\left(x+x_{1}\right)\left[\frac{3}{\left(x+2 x_{1}\right) x_{1}^{3}}-\frac{7\left(2 x_{1}^{2}-1\right)}{4 x_{1}^{4}\left(x+2 x_{1}\right)^{2}}\right]\right\},
\end{aligned}
$$

Consequently, to lowest order in $|x| \ll 1$ and $\left|x_{1}\right| \ll 1$ the averaged energy density will be

$$
\left\langle\hat{\rho}_{\mathrm{gw}}\right\rangle=-\frac{5}{6} \frac{1}{a^{2}} \int \frac{d \ln k}{2 \pi^{2} \tau_{1}^{4}} k^{2},
$$

which is the result reported in Eq. (4.9). Along similar lines the averaged pressure density can be obtained:

$$
\begin{aligned}
\left\langle\hat{p}_{\mathrm{gw}}\right\rangle= & \frac{1}{3 a^{4}} \int \frac{k^{4} d \ln k}{2 \pi^{2}}\left\{\frac{1}{2}\left[2-\frac{5}{\left(x+2 x_{1}\right)^{2}}\right]\left(1+\frac{1}{2 x_{1}^{4}}\right)+\frac{1}{4 x_{1}^{4}}\left(12-\frac{5}{\left(x+2 x_{1}\right)^{2}}\right)\right. \\
\left.\times\left[\left(2 x_{1}^{2}-1\right) \cos 2\left(x+x_{1}\right)+2 x_{1} \sin 2\left(x+x_{1}\right)\right]-\frac{5}{2\left(x+2 x_{1}\right) x_{1}^{4}}\left[\left(2 x_{1}^{2}-1\right) \sin 2\left(x+x_{1}\right)-2 x_{1} \cos 2\left(x+x_{1}\right)\right]\right\}, & \\
\left\langle\hat{\mathcal{P}}_{\mathrm{gw}}\right\rangle-\left\langle\hat{p}_{\mathrm{gw}}\right\rangle= & \frac{8}{3 a^{4}} \int \frac{d^{3} k}{(2 \pi)^{3}}\left[-\frac{\left(2 x_{1}^{2}-1\right)}{2 x_{1}^{4}} \sin 2\left(x+x_{1}\right)+\frac{1}{x_{1}^{3}} \cos 2\left(x+x_{1}\right)-\frac{1}{\left(x+2 x_{1}\right)}\left(1+\frac{1}{2 x_{1}^{4}}\right)\right. \\
& \left.\quad-\frac{\left(2 x_{1}^{2}-1\right)}{2 x x_{1}^{4}} \cos 2\left(x+x_{1}\right)-\frac{1}{\left(x+2 x_{1}\right) x_{1}^{3}} \sin 2\left(x+x_{1}\right)\right]
\end{aligned}
$$

implying, to lowest order in $|x| \ll 1$ and $\left|x_{1}\right| \ll 1$,

$$
\left\langle\hat{p}_{\mathrm{gw}}\right\rangle=\frac{7}{6} \frac{1}{a^{2}} \int \frac{d \ln k}{2 \pi^{2} \tau_{1}^{4}} k^{2}, \quad\left\langle\hat{\mathcal{P}}_{\mathrm{gw}}\right\rangle-\left\langle\hat{p}_{\mathrm{gw}}\right\rangle=-\frac{8}{9} \frac{1}{a^{2}} \int \frac{d \ln k}{2 \pi^{2} \tau_{1}^{4}} k^{2},
$$

which are the results reported in Eqs. (4.10) and (4.11).

Instead of using the energy-momentum pseudotensor derived from the quadratic corrections to the Einstein tensor, we could use the energy and pressure densities proposed in [38,39] (see also [6]). In this second case the expectation values of the energy and pressure density become: 


$$
\begin{aligned}
\left\langle\hat{\rho}_{\mathrm{gw}}\right\rangle= & \frac{1}{a^{4}} \int \frac{k^{4}}{2 \pi^{2}} d \ln k\left\{\frac { 1 } { 2 } \left[2+\frac{1}{\left(x+2 x_{1}\right)^{2}}\left(1+\frac{1}{2 x_{1}^{4}}\right)+\cos 2\left(x+x_{1}\right)\left[\frac{\left(2 x_{1}^{2}-1\right)}{4 x_{1}^{4}\left(x+2 x_{1}\right)^{2}}-\frac{1}{x_{1}^{3}\left(x+2 x_{1}\right)}\right]\right.\right. \\
& \left.+\sin 2\left(x+x_{1}\right)\left[\frac{\left(2 x_{1}^{2}-1\right)}{2 x_{1}^{4}\left(x+2 x_{1}\right)}+\frac{1}{2 x_{1}^{3}\left(x+2 x_{1}\right)^{2}}\right]\right\}, \\
\left\langle\hat{p}_{\mathrm{gw}}\right\rangle= & \frac{1}{a^{4}} \int \frac{k^{4}}{2 \pi^{2}} d \ln k\left\{\frac{1}{2}\left(1+\frac{1}{2 x_{1}^{4}}\right)\left[\frac{2}{3}+\frac{1}{\left(x+2 x_{1}\right)^{2}}\right]+\cos 2\left(x+x_{1}\right)\left[\frac{2 x_{1}^{2}-1}{4 x_{1}^{4}}\left(\frac{1}{\left(x+2 x_{1}\right)^{2}}-\frac{4}{3}\right)-\frac{1}{x_{1}^{3}\left(x+2 x_{1}\right)}\right]\right. \\
& \left.+\sin 2\left(x+x_{1}\right)\left[\frac{1}{2 x_{1}^{3}}\left(\frac{1}{\left(x+2 x_{1}\right)^{2}}-\frac{4}{3}\right)+\frac{2 x_{1}^{2}-1}{2 x_{1}^{4}\left(x+2 x_{1}\right)}\right]\right\}
\end{aligned}
$$

If we now expand the previous expressions for $\left|x_{1}\right| \ll 1$ and for $|x| \ll 1$ we get to the results reported in Eqs. (4.14) and (4.15).

In the following we are going to cross-check some of the results obtained in the case of the $\sigma$ parametrization but working directly in the conformal time coordinate. It should be clear that the specific parametrization is not crucial to obtain a given result. However, it can happen that, for practical reasons, a given parametrization has to be preferred since it leads either to more transparent or to swifter results. The idea will be here to solve the evolution of the mode functions exactly but keeping an approximate form of the background in the post-bounce and in the prebounce region. In the conformal time parametrization the action given in Eq. (1.4) reads

$$
S_{\mathrm{gw}}=\frac{1}{2} \int d^{3} x d \tau a^{2}\left[\left(\partial_{\tau} h\right)^{2}-a^{2}\left(\partial_{m} h\right)^{2}\right],
$$

whose associate Hamiltonian is

$$
H_{\mathrm{gw}}=\frac{1}{2} \int d^{3} x\left[\Pi^{2}+a^{2}\left(\partial_{m} h\right)^{2}\right],
$$

with canonical momentum given as $\Pi=a^{2} h^{\prime}$. Therefore in this case the mode functions obey

$$
\tilde{f}_{k}^{\prime \prime}+2 \mathcal{H} \tilde{f}_{k}^{\prime}+k^{2} \tilde{f}_{k}=0, \quad \tilde{g}_{k}=a^{2} \tilde{f}_{k}^{\prime} .
$$

Consider, for instance, the case given in Eqs. (2.25), (2.26), and (2.27). The form of the mode functions before the bounce (i.e. for $\tau<-\tau_{1}$ ) will then be

$$
\begin{aligned}
& \tilde{f}_{k}(\tau)=\frac{\sqrt{\tau_{1}} \bar{\pi}}{\sqrt{2} a_{-}} e^{i \pi / 4} H_{0}^{(1)}(-x), \\
& \tilde{g}_{k}(\tau)=-a^{2}(\tau) k \sqrt{\tau_{1}} e^{i \pi / 4} \sqrt{\frac{\pi}{2}} H_{1}^{(1)}(-x),
\end{aligned}
$$

where, as in the previous example, $x=k \tau ; H_{\nu}^{1}(z)$ and $H_{\nu}^{(2)}(z)$ will denote, in the following, the Hankel functions of index $\nu$ and argument $z$ [62,63]. For $\tau>\tau_{1}$, recalling Eq. (2.26), the evolution of the mode functions can be written as:

$$
\begin{aligned}
\tilde{F}_{k}(\tau)= & \frac{\sqrt{\pi}}{\sqrt{2}} \frac{\sqrt{\tau_{1}}}{a_{+}}\left[c_{+}(k) e^{i \pi / 4} H_{0}^{(1)}(x)+c_{-}(k) e^{-i \pi / 4} H_{0}^{(2)}(x)\right], \\
\tilde{G}_{k}(\tau)= & -a^{2}(\tau) \frac{k \sqrt{\tau_{1}}}{a_{+}} \sqrt{\frac{\pi}{2}}\left[c_{+}(k) e^{i \pi / 4} H_{1}^{(1)}(x)\right. \\
& \left.+c_{-}(k) e^{-i \pi / 4} H_{1}^{(2)}(x)\right] .
\end{aligned}
$$

It is now instructive to pretend that the analytical form of the mode functions is unavailable in the intermediate region, i.e. $-\tau_{1}<\tau<\tau_{1}$. Therefore let us try to excise a thin region around the bounce and compute the mixing coefficients by assuming

$$
\tilde{f}_{k}\left(-\tau_{1}\right)=\tilde{F}_{k}\left(\tau_{1}\right), \quad \tilde{g}_{k}\left(-\tau_{1}\right)=\tilde{G}_{k}\left(\tau_{1}\right) .
$$

This allows to determine

$c_{+}(k)=-i \frac{\pi}{4} x_{1}\left[H_{1}^{(2)}\left(x_{1}\right) H_{0}^{(1)}\left(x_{1}\right)+H_{0}^{(2)}\left(x_{1}\right) H_{1}^{(1)}\left(x_{1}\right)\right]$,

$c_{-}(k)=-\frac{\pi}{2} x_{1} H_{0}^{(1)}\left(x_{1}\right) H_{1}^{(1)}\left(x_{1}\right)$,

Notice that from the previous formulas it is possible to obtain, after some trivial algebra, that $\left|c_{+}(k)\right|^{2}-$ $\left|c_{-}(k)\right|^{2}=1$. Recalling now that $[62,63]$

$$
H_{0}^{(1)}\left(x_{1}\right) \sim i \frac{2}{\pi} \ln x_{1}, \quad H_{1}^{(1)}\left(x_{1}\right) \sim-\frac{2 i}{\pi x_{1}}
$$

we find that for $x_{1} \ll 1$

$$
c_{-}(k) \sim-\frac{2}{\pi} \ln x_{1}
$$

Now this result is (qualitatively) similar to the one obtained, by a totally different approach, in Eqs. (3.16), (3.17), and (3.30). By doing a more careful comparison it is possible to show that the method discussed in Sec. III of the present paper is the most accurate, also from a conceptual point of view. However, the path followed in the present appendix is able to reproduce the gross features of the mixing and, therefore, can be used for semiquantitative estimates.

It is interesting to notice that, within this approach, the results of the effective barotropic indices (numerically obtained in the text) can be checked. For instance, in the approach of [38,39] the energy and pressure densities 
before the bounce become, in the present example

$$
\begin{aligned}
\left\langle\hat{\rho}_{\mathrm{gw}}\right\rangle= & \frac{1}{a^{4}} \int \frac{k^{4}}{2 \pi^{2}} d \ln k\left\{\frac { \pi } { 4 } ( - x ) \left[J_{1}(-x)^{2}+Y_{1}(-x)^{2}\right.\right. \\
& \left.\left.+J_{0}(-x)^{2}+Y_{0}(-x)^{2}\right]\right\} \\
\left\langle\hat{p}_{\mathrm{gw}}\right\rangle= & \frac{1}{a^{4}} \int \frac{k^{4}}{2 \pi^{2}} d \ln k\left\{\frac{\pi}{4}(-x)\left[J_{1}(-x)^{2}+Y_{1}(-x)^{2}\right]\right. \\
& \left.-\frac{\pi}{12}(-x)\left[J_{0}(-x)^{2}+Y_{0}(-x)^{2}\right]\right\}
\end{aligned}
$$

where $J_{\nu}(z)$ and $Y_{\nu}(z)$ are the Bessel functions of order $\nu$ and argument $z$ [62,63]. In the small argument limit, from Eqs. (C27) we do get:

$$
\left\langle\hat{p}_{\mathrm{gw}}\right\rangle \simeq\left\langle\hat{\rho}_{\mathrm{gw}}\right\rangle=\frac{1}{a^{4}} \int \frac{k^{4}}{2 \pi^{2}} d \ln k\left(-\frac{1}{\pi x}\right),
$$

which is one of the results discussed in Sec. IV. Both $\left\langle\rho_{\mathrm{gw}}\right\rangle$ and $\left\langle p_{\text {gw }}\right\rangle$ scale as $a^{-6}$ (since $a(\tau) \sim \sqrt{-\bar{\tau}}$ ) as it is consistent with the equation of state $\left\langle\hat{\rho}_{\mathrm{gw}}\right\rangle \simeq\left\langle\hat{p}_{\mathrm{gw}}\right\rangle$.

The same analysis can be preformed when the energymomentum pseudotensor is obtained from the quadratic corrections to the Einstein tensor. The result is

$$
\begin{aligned}
\left\langle\hat{\rho}_{\mathrm{gw}}\right\rangle & =\frac{1}{a^{4}} \int \frac{k^{4}}{2 \pi^{2}} d \ln k\left\{\pi\left[J_{0}(-x) J_{1}(-x)+Y_{0}(-x) Y_{1}(-x)\right]-\frac{\pi}{4}\left[J_{0}(-x)^{2}+J_{1}(-x)^{2}+Y_{0}(-x)^{2}+Y_{1}(-x)^{2}\right]\right\}, \\
\left\langle\hat{p}_{\mathrm{gw}}\right\rangle & =\frac{1}{a^{4}} \int \frac{k^{4}}{2 \pi^{2}} d \ln k\left\{\pi\left[J_{0}(-x) J_{1}(-x)+Y_{0}(-x) Y_{1}(-x)\right]-\frac{\pi}{4} x\left[J_{0}(-x)^{2}+Y_{0}(-x)^{2}+J_{1}(-x)^{2}+Y_{1}(-x)^{2}\right]\right\}, \\
\left\langle\hat{\mathcal{P}}_{\mathrm{gw}}\right\rangle-\left\langle\hat{p}_{\mathrm{gw}}\right\rangle & =\frac{1}{a^{4}} \int \frac{k^{4}}{2 \pi^{2}} d \ln k 4 \pi\left[J_{0}(-x) J_{1}(-x)+Y_{0}(-x) Y_{1}(-x)\right] .
\end{aligned}
$$

Let us now expand the previous expressions for $|x| \ll 1$. The result is:

$$
\begin{aligned}
\left\langle\hat{\rho}_{\mathrm{gw}}\right\rangle & =\frac{1}{a^{4}} \int \frac{k^{4}}{2 \pi^{2}} d \ln k\left[-\frac{1}{\pi x}(1-4 \gamma+4 \ln 2-4 \ln (-x))\right], \\
\left\langle\hat{p}_{\mathrm{gw}}\right\rangle & =\frac{1}{a^{4}} \int \frac{k^{4}}{2 \pi^{2}} d \ln k \frac{5}{3 \pi x}, \\
\left\langle\hat{\mathcal{P}}_{\mathrm{gw}}\right\rangle-\left\langle\hat{p}_{\mathrm{gw}}\right\rangle & =\frac{1}{a^{4}} \int \frac{k^{4}}{2 \pi^{2}} d \ln k \frac{2}{\pi x}[2 \gamma-2 \ln 2+2 \ln (-x)] .
\end{aligned}
$$

where $\gamma$ is the Euler-Mascheroni constant [62,63]. From Eqs. (C30) it is possible to obtain that in this parametrization of the energy-momentum pseudotensor, indeed, logarithmic corrections to the effective barotropic index arise, as pointed out in Sec. IV. From the examples reported here it is clear that also the conformal time parametrization can be employed for the self-consistent analysis of backreaction effects. Indeed, in Sec. V, explicit numerical integrations have been reported also in the $\tau$ parametrization. It is however true that, in the context of bouncing models, the $\sigma$ coordinate allows to obtain the wanted results much faster both analytically and numerically.

[1] L. P. Grishchuk, Zh. Eksp. Teor. Fiz. 67, 825 (1974) [Sov. Phys. JETP 40, 409 (1975)].

[2] L. P. Grishchuk, Ann. N.Y. Acad. Sci. 302, 439 (1977).

[3] A. A. Starobinsky, JETP Lett. 30, 682 (1979).

[4] L. P. Grishchuk and M. Solokhin, Phys. Rev. D 43, 2566 (1991).

[5] M. Gasperini and M. Giovannini, Phys. Lett. B 282, 36 (1992); Phys. Rev. D 47, 1519 (1993).

[6] V. Sahni, Phys. Rev. D 42, 453 (1990).

[7] V. A. Rubakov, M. V. Sazhin, and A. V. Veryaskin, Phys. Lett. B 115, 189 (1982).

[8] R. Fabbri and M.D. Pollock, Phys. Lett. B 125, 445 (1983).

[9] L.F. Abbott and M. B. Wise, Nucl. Phys. B244, 541 (1984).
[10] G. Veneziano, Phys. Lett. B 265, 287 (1991).

[11] M. Gasperini and G. Veneziano, Phys. Rep. 373, 1 (2003).

[12] R. Brustein, M. Gasperini, M. Giovannini, and G. Veneziano, Phys. Lett. B 361, 45 (1995).

[13] M. Galluccio, M. Litterio, and F. Occhionero, Phys. Rev. Lett. 79, 970 (1997).

[14] M.P. Infante and N. Sanchez, Phys. Rev. D 61, 083515 (2000).

[15] M. Giovannini, Phys. Rev. D 58, 083504 (1998).

[16] Ya. B. Zeldovich and A. A. Starobinsky, Zh. Eksp. Teor. Fiz. 61, 2161 (1971) [Sov. Phys. JETP 34, 1159 (1972)].

[17] L. H. Ford, Phys. Rev. D 35, 2955 (1987).

[18] N.D. Birrel and P.C. W. Davies, J. Phys. A 13, 2109 (1980). 
[19] N.D. Birrel and P.C.W. Davies, Quantum Fields in Curved Space (Cambridge University Press, Cambridge,UK, 1982).

[20] P. J.E. Peebles and A. Vilenkin, Phys. Rev. D 59, 063505 (1999).

[21] M. Giovannini, Phys. Rev. D 60, 123511 (1999); Classical Quantum Gravity 16, 2905 (1999).

[22] M. Giovannini, Phys. Rev. D 67, 123512 (2003).

[23] H. Tashiro, T. Chiba, and M. Sasaki, Classical Quantum Gravity 21, 1761 (2004).

[24] D. Babusci and M. Giovannini, Phys. Rev. D 60, 083511 (1999).

[25] F. Y. Li, M.X. Tang, and D. P. Shi, Phys. Rev. D 67, 104008 (2003).

[26] A. M. Cruise, Classical Quantum Gravity 17, 2525 (2000).

[27] P. Bernard, G. Gemme, R. Parodi, and E. Picasso, Rev. Sci. Instrum. 72, 2428 (2001).

[28] V. Sahni, M. Sami, and T. Souradeep, Phys. Rev. D 65, 023518 (2002).

[29] M. Sami and V. Sahni, Phys. Rev. D 70, 083513 (2004).

[30] M. Sami, N. Dadhich, and T. Shiromizu, Phys. Lett. B 568, 118 (2003).

[31] M. Yahiro, G. J. Mathews, K. Ichiki, T. Kajino, and M. Orito, Phys. Rev. D 65, 063502 (2002).

[32] L. D. Landau and E. M. Lifshitz, The Classical Theory of Fields (Addison-Wesley/Pergamon Press, New York, 1971).

[33] R. Isaacson, Phys. Rev. 166, 1263 (1968).

[34] R. Isaacson, Phys. Rev. 166, 1272 (1968).

[35] L. R. Abramo, R. Brandenberger, and V. Mukahanov, Phys. Rev. D 56, 3248 (1997).

[36] L. R. Abramo, Phys. Rev. D 60, 064004 (1999).

[37] S. V. Babak and L. P. Grishchuk, Phys. Rev. D 61, 024038 (2000).

[38] L. H. Ford and L. Parker, Phys. Rev. D 16, 1601 (1977).

[39] L. H. Ford and L. Parker, Phys. Rev. D 16, 245 (1977).

[40] K. A. Meissner and G. Veneziano, Mod. Phys. Lett. A 6, 3397 (1991).

[41] K. A. Meissner and G. Veneziano, Phys. Lett. B 267, 33 (1991).
[42] M. Gasperini, J. Maharana, and G. Veneziano, Nucl. Phys. B472, 349 (1996).

[43] J. Maharana and J.H. Schwarz, Nucl. Phys. B390, 3 (1993).

[44] J. Maharana, Phys. Lett. B 296, 65 (1992).

[45] M. Gasperini, M. Giovannini, and G. Veneziano, Phys. Lett. B 569, 113 (2003).

[46] M. Gasperini, M. Giovannini, and G. Veneziano, Nucl. Phys. B694, 206 (2004).

[47] M. Gasperini and G. Veneziano, Astropart. Phys. 1, 317 (1993).

[48] M. Giovannini, Classical Quantum Gravity 21, 4209 (2004).

[49] N. Deruelle and A. Streich, Phys. Rev. D 70, 103504 (2004).

[50] N. Deruelle, gr-qc/0404126.

[51] C. Gordon and N. Turok, Phys. Rev. D 67, 123508 (2003).

[52] J.c. Hwang and H. Noh, Phys. Rev. D 66, 084009 (2002).

[53] G.F.R. Ellis and M.S. Madsen, Classical Quantum Gravity 8, 667 (1991).

[54] G. F. R. Ellis and R. Maartens, Classical Quantum Gravity 21, 223 (2004).

[55] L. Parker and S. A. Fulling, Phys. Rev. D 7, 2357 (1973).

[56] A. Starobinsky, Sov. Astron. Lett. 4, 82 (1978).

[57] J.D. Barrow and R. A. Matzner, Phys. Rev. D 21, 336 (1980).

[58] C. I. Kuo and L. H. Ford, Phys. Rev. D 47, 4510 (1993).

[59] B. Hu and L. Parker, Phys. Lett. A 63, 217 (1977).

[60] W. H. Press, S. A. Teutolsky, W. T. Vetterling, and B.P. Flannery, Numerical Recipes in Fortran 77 (Cambridge University Press, Cambridge, UK, 1992), 2nd ed., p. 731.

[61] V. Bozza, M. Giovannini, and G. Veneziano, J. Cosmol. Astropart. Phys. 05 (2003) 001; M. Giovannini, Classical Quantum Gravity 20, 5455 (2003).

[62] M. Abramowitz and I. A. Stegun, Handbook of Mathematical Functions (Dover, New York, 1972).

[63] A. Erdelyi, W. Magnus, F. Obehettinger, and F. Tricomi, Higher Trascendental Functions (Mc Graw-Hill, New York, 1953). 